\title{
Spatiotemporal expression of FRIGIDA modulate flowering time in Arabidopsis thaliana
}

Xiangxiang Kong ${ }^{1}$, Jinjie Zhao ${ }^{1}$, Landi Luo $^{2}$, Qian Chen ${ }^{1}$, Guanxiao Chang ${ }^{1,3}$, Jinling Huang ${ }^{1,3}$, Yongping Yang ${ }^{1,2 *}$, Xiangyang $\mathrm{Hu}^{4, *}$

${ }^{1}$ Key Laboratory for Plant Diversity and Biogeography of East Asia, Kunming Institute of Botany, Chinese Academy of Sciences, Kunming 650201, China

${ }^{2}$ School of Ecology and Environmental Science, Yunnan University, Kunming 650504, China

${ }^{3}$ Institute of Plant Stress Biology, State Key Laboratory of Cotton Biology, Henan University, 475001, Kaifeng

${ }^{4}$ Shanghai Key Laboratory of Bio-Energy Crops, Plant Science Center, School of Life Sciences, Shanghai University, Shanghai, 200444, China

\section{Corresponding author:}

Xiangyang Hu, Tel: +86-21-66133511, E-mail: huxiangyang@ shu.edu.cn

Yongping Yang, Tel: +86-553-68625531, E-mail: yongyp@mail.kib.ac.cn

\section{Emails:}

Xiangxiang Kong: kongxiangxiang@mail.kib.ac.cn

Jinjie Zhao: zhaojinjie@mail.kib.ac.cn

Landi Luo: landiluo@126.com

Qian Chen: chenqian@mail.kib.ac.cn

Guanxiao Chang: changguanxiao@henu.edu.cn

Jinling Huang: huangj@ecu.edu

Yongping Yong: yangyp@mail.kib.ac.cn

The date of submission: 12 November 2018; The of number Figures: 9; the word count: 6420 .

Running Title: Spatial-temporal effect of FRI on flowering 
bioRxiv preprint doi: https://doi.org/10.1101/467613; this version posted November 11,2018 . The copyright holder for this preprint (which was not certified by peer review) is the author/funder. All rights reserved. No reuse allowed without permission.

\section{Highlight:}

Root FRIDIGA activated the novel targets $M A F 4 / 5$ to delay flowering; Temporal expressing FRIGIDA at as early as pro-embryo stage is efficient to delay flowering. 


\begin{abstract}
FRIGIDA (FRI) as the major regulator of flowering time in Arabidopsis accessions can activate its target FLOWERING LOCUS C (FLC) to delay flowering before vernalization. Besides FLC, other FRI targets also exist in Arabidopsis. Although leaves sense environmental cues to modulate flowering time, it is not known if roots also regulate the floral transition. In this study, we investigated the spatiotemporal effect of FRI on flowering time. Local expression of $F R I$ in the phloem and leaves activated FLC to delay flowering. Furthermore, we found that local expression of FRI in the roots also delayed flowering by activating other targets MADS AFFECTING FLOWERING4 (MAF4) and MAF5 in the roots. Graft and genetic experiments revealed that the spatial expression of $F R I$ in the root might generate a mobile signal, which is transmitted from roots to shoot and antagonizes the FT signal to delay flowering. Specifically expressing FRI in the embryo efficiently delayed flowering, even expressing $F R I$ as early as pro-embryo stage is enough to upregulate $F L C$ expression to delay flowering. Together, our findings confirm the spatiotemporal effect of FRI on delaying flowering, and propose that root tissue also perceives the flowering signal to fine-tune the flowering time through MAF4/5 as novel targets of FRI.
\end{abstract}

Keyword: FRIGIDA; spatial-temporal expression; flowering; Arabidopsis 


\section{Introduction}

Flowering at the appropriate time is fundamental to reproductive success of plants and their adaptation to environmental stress. As such, plants have evolved accurate mechanisms to initiate flowering in response to exogenous environmental cues or endogenous signals (Jaeger et al., 2006; Kim et al., 2009; Amasino and Michaels, 2010). Many plant species require a long period of cold treatment (i.e., vernalization) before flowering (Kim et al., 2009). FRIGIDA (FRI) is a major regulator of flowering time in various Arabidopsis thaliana accessions (Johanson et al., 2000). The winter-annual (late flowering) and rapid-cycling (early flowering) accessions are often distinguished by allelic variation at FRI and Flowering Locus C (FLC) (Shindo et al., 2005; Shindo et al., 2006). FRI encodes a novel protein with two potential coiled-coil domains that activates the expression of $F L C$, which in turn encodes a MADS box transcriptional factor that quantitatively inhibits the floral transition through repressing the flowering pathway integrators FLOWERING LOCUS T (FT) and SUPPRESSOR OF OVEREXPRESSION OF CONSTANS1 (SOC1) in Arabidopsis (Johanson et al., 2000; Michaels, 2004; Schmitz, 2007).

Although FRI was isolated two-decade ago, its biochemical function and the mechanism underlying flowering regulation are largely unknown. FRI acts as a scaffold protein that interacts with FRL1, SUF4, FLX, FES1, UBC1 and CBP20 to form a transcription activator complex, which recruits chromatin modification factors, such as the SWRI complex and SET2 homolog, to epigenetically modify the histone methylation level at the FLC locus (Choi et al., 2011; Li et al., 2018). Besides FLC, other five FLC homologues, including FLOWERING LOCUS M (FLM)/MADS AFFECTING FLOWERING 1, and MAF2-MAF5, also modulate flowering time (Ratcliffe et al., 2003; Scortecci et al., 2003; Gu et al., 2009; Kim et al., 2010). MAF4 can directly interact with other FLC homologues proteins to repress FT expression (Gu et al., 2013). MAF4 and MAF5 is rather specific to preventing a precocious vernalization response (Kim and Sung, 2013). Nevertheless, the underlying mechanisms remain to be discovered.

In many plants, the transition from vegetative to reproductive development is controlled by 
environmental cues, such as day length and temperature (Amasino and Michaels, 2010). The environmental signal is usually perceived by the leaves, while flowers develop from primordia formed on the flanks of the SAM (shoot apical meristem). Ectopic expression of FLC in the phloem of leaves can reduce SOC1 and FT mRNA levels to delay flowering (Searle et al., 2006). Unlike these findings in leaves, previous studies show that ectopic expression of $F L C$ in the root did not efficiently delay the flowering time (Searle et al., 2006), and ectopic expression of FT in the root also could not efficiently promote flowering time (Abe et al., 2005), casting doubt on the contribution of the root to modulating flowering time. However, other lines of evidence imply that the root tissue might regulate flowering time. For example, in white mustard (Sinapis alba), a shoot-to-root-to-shoot physiological loop driving sugar and cytokinin fluxes is essential for flowering (Bernier et al., 1993). Data mining of public microarray experiments showed that about 200 genes associated with flowering time are expressed in the root (Bouche et al., 2016). Thus, studying the spatial and temporal effects of FRI on flowering time should reveal at which developmental stage FRI epigenetically regulates gene expression to delay flowering and whether root tissue modulates flowering time.

In this study, we systemically investigated the effect of various spatial and temporal expression patterns of FRI on flowering time in Arabidopsis. We found that expression of FRI in the leaves or phloem activated FLC transcription in a cell-autonomous manner and thereby delayed flowering. In contrast to FLC, which does not delay flowering when spatially expressed in root tissue (Searle $e t$ $a l ., 2006)$, we found that ectopic expression of $F R I$ in the roots also delayed flowering by activating the transcription of MAF4 and MAF5, and subsequently suppressed the downstream $F T$ signal depending on downstream mobile signals, such as antiflorigen to delay flowering. Furthermore, expressing FRI only during embryonic development or in the young seedling stage using temporal-specific promoters also efficiently delayed flowering time, and was accompanied by high levels of histone methylation at the FLC locus. Based on our data, we propose that, in addition to leaves, which are believed to be the main sensors of the environmental signal, roots also play critical roles in modulating flowering time. Thus, our data provide novel insight into the molecular mechanism underlying FRI-mediated regulation of flowering time. 


\section{Materials and methods}

\section{Plant materials and growth conditions}

Wild-type Arabidopsis thaliana $(\mathrm{Col})$ plants were used in this study. $\mathrm{Col}$ seeds were sown in plastic pots under a 16-h light/8-h dark photoperiod. For vernalization, seeds were germinated and pre-grown for 7 days under standard conditions (16 h light/ $8 \mathrm{~h}$ darkness, $\left.22^{\circ} \mathrm{C}\right)$. The plants were transferred to cold conditions $\left(8 \mathrm{~h}\right.$ light $/ 16 \mathrm{~h}$ darkness, $\left.4^{\circ} \mathrm{C}\right)$ for 4 weeks, and then returned to standard conditions. The time to flowering was determined as the total number of rosette leaves when the floral bolt was $1 \mathrm{~cm}$ high (Sheldon et al., 2008; Hu et al., 2014).

\section{Development of transgenic lines}

To construct the 35S:GFP-FRI plasmid, the full-length cDNA coding region of FRI was amplified and cloned downstream of the GFP fluorescence marker in the $p E G A D$ vector. To construct the 35S:GUS-FRI plasmid, the coding region of GFP of 35S:GFP-FRI was replaced by the GUS fragment. To generate the FRI::GUS-FRI construct, the promoter FRI with $2.1 \mathrm{~kb}$ of genomic sequence were amplified from winter-annual accession $H 51$, and the $35 \mathrm{~S}$ promoter of the $35 S:: F R I-G U S$ construct was replaced with the FRI promoter. To drive FRI expression by the spatiotemporal-specific promoters, the promoters SUC2, ML1, KNAT, RolC, TobRB7, LEC2, and EM1 were amplified, and the $35 \mathrm{~S}$ promoter of the $35 S:: F R I-G U S$ construct was replaced with the above promoters, respectively. To construct the TobRB7::MAF4 or TobRB7::MAF5 plasmid, the full-length cDNA of MAF4 or MAF5 was amplified and replaced the GFP fluorescence marker in the $p E G A D$ vector, then the $35 S$ promoter was replaced with the TobRB7 promoter. To generate the inducible ProER8:GUS-FRI plasmid, the coding region of GUS-FRI was amplified and inserted into the $p E R 8$ vector. These constructs were directly transformed into Agrobacterium tumefaciens GV3101, and the modified floral dip method was performed to generate the corresponding transgenic plants in the Col background. The transgenic seeds were screened on half-strength Murashige and Skoog medium containing $10 \mathrm{mg} / \mathrm{L}$ Basta or $35 \mathrm{mg} \mathrm{L}{ }^{-1}$ Hygromycin. The T3 homologous transgenic seeds were used in our experiments. All of the primer sequences are listed in 
Supplemental Table 1.

\section{RNA extraction and quantitative RT-PCR}

Total RNA was extracted using Trizol reagent (Invitrogen). Quantitative real-time PCR (qRT-PCR) was performed as described previously. Briefly, first-strand cDNA was synthesized from $1.5 \mu \mathrm{g}$ DNAse-treated RNA in a 20- $\mu \mathrm{L}$ reaction volume using M-MuLV Reverse Transcriptase (Invitrogen) with oligo $(\mathrm{dT})_{18}$ primer. RT-PCR was performed using 2 XSYBR Green I Master on a Roche LightCycler 480 real-time PCR machine, according to the manufacturer's instructions. At least three biological replicates for each sample were used for the RT-PCR analysis, and at least three technical replicates were analyzed for each biological replicate. The Actin gene was used as a control. Gene-specific primers used to detect transcripts are listed in Supplemental Table 1.

\section{ChIP assay}

ChIP was performed largely as described previously (Hu et al., 2014). Immunoprecipitation was performed with acetylated H3 (1:1000; Millipore) and trimethyl H3K4 (1:500; Millipore) or trimethyl H3K27 (1:500; Millipore) antibody Both immunoprecipitated DNA and input DNA were analyzed by real-time PCR. Primers and PCR detection of FLC regions were as described (in Supplemental Table 1). Data from ChIP experiments are expressed as means \pm SD of three biological replicates.

\section{Protoplast transient assay}

Rosette leaves of Arabidopsis Col plants that had been grown for 4 weeks under long-day conditions (16 h light/8 h darkness) were sampled for the isolation and transformation of protoplasts as described (Yoo et al., 2007). All of the plasmid DNA for the protoplast transformation was prepared by the $\mathrm{CsCl}$ gradient method. Twelve hours after transformation, the protoplasts were observed. Fluorescence of the GFP chimeric gene was detected using an Olympus FluoView confocal microscope with excitation and emission filters of 450-490 nm and 520-560 nm, respectively. 


\section{Histochemical analysis of GUS activity}

Whole tissues were vacuum-infiltrated in GUS-staining buffer $[0.5 \mathrm{mM} \mathrm{NaPO}$ ( $\mathrm{pH} 7.0), 10 \mathrm{mM}$ EDTA, $1 \mathrm{mM}$ potassium ferrocyanide, $1 \mathrm{mM}$ potassium ferricyanide, $1 \mathrm{mM}$ 5-bromo-4-chloro-3-indolyl-3-d-glucuronic acid (X-Gluc), and 0.1\% Triton X-100], incubated at $37^{\circ} \mathrm{C}$, and then destained in $70 \%$ ethanol. Cross-sections of leaf and stem tissues were prepared using a razor blade followed by staining as described above. For cross-sections of cotyledons, GUS-stained seedlings were fixed (50\% ethanol, 5\% acetic acid, 3\% formaldehyde), dehydrated in a graded ethanol series $(70 \%, 96 \%$, and 100\%; 2 h each), embedded in Technovit 7100 (Heraeus Kulzer), according to the manufacturer's instructions, and sectioned using a microtome.

\section{Immunoblot assay}

Total proteins were prepared by grinding seedlings on ice in extraction buffer (50 mM Tris, 5\%

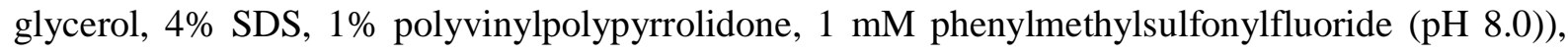
followed by centrifugation at $4^{\circ} \mathrm{C}$ and $14,000 \mathrm{~g}$ for $15 \mathrm{~min}$. A $15-\mu \mathrm{g}$ aliquot of protein was separated by electrophoresis on a 12\% SDS-polyacrylamide gel and blotted onto polyvinylidene difluoride membranes, which were then probed with the appropriate primary anti-GFP (1:3000, Clontech) or anti-actin (1:1000, Sigma-Aldrich) antibody and horseradish peroxidase-conjugated goat anti-mouse secondary antibody (1:3000, Promega). Signals were detected using the ONE-HOUR IP-Western Kits (Cat. L00232, Genescript).

\section{Grafting methods}

The general grafting conditions and procedures were performed as reported previously (Turnbull et al., 2002). Briefly, young seedlings with the cotyledons expanded were used as the scions and rootstocks. We used a sapphire/diamond knife to remove the cotyledons from all seedlings, and cut the scion and rootstock seedlings cleanly across the hypocotyl just below the cotyledon stumps. The rootstock and scion were well-matched for size where the cut ends could be pressed very closely together. After all grafts were complete, the grafted plants were placed in petri dishes in the growth room for 3-5 days, and then were moved to soil and grown until flowering, and the flowering time was scored. 


\section{Accession numbers}

The T-DNA insertion lines were used in this study: maf4-1(salk_028506), maf4-1(salk_095092), ft-10 (CS9869) and flc-3 (Germplasm:1008704442)

\section{Results}

\section{Constitutive expression of FRI delays flowering time}

FRI upregulates FLC transcription and thereby delays flowering time (Geraldo et al., 2009; Choi et $a l ., 2011)$. To decipher the role of $F R I$ in controlling flowering time, we investigated the expression pattern of FRI using constructs in which the GUS reporter marker is driven by the endogenous $F R I$ promoter. As shown in Fig. 1A, GUS staining could be observed in the young embryo and cotyledon, and in the leaves, vasculature, meristem, and roots of 2-week-old seedling. We then generated a construct containing $F R I$ fused to green fluorescence protein $(G F P)$ under the control of a cauliflower mosaic virus (CaMV) 35S promoter (termed $35 S:: G F P-F R I)$. When $35 S:: G F P-F R I$ was transiently expressed in Nicotiana benthamiana leaves or in protoplasts derived from Arabidopsis Col leaves, fluorescence was detected only in the nucleus (Fig. 1B), which is consistent with our previous results that FRI is mainly localized in the nucleus (Hu et al., 2014).

We then generated transgenic line expressing $35 S: \because G F P-F R I$ and examined GFP-FRI expression in three individual T3 line. Immunoblot analysis revealed that all of these lines accumulated high levels of GFP-FRI (Fig. 1C). Consistent with the strong accumulation of GFP-FRI in these lines, these lines showed delayed flowering compared with wild-type Col in long days (LD, 16-h light/8-h dark) and short days (SD, 8-h light/16-h dark) (Fig. 1C\&D, Fig. S1). However, vernalization treatment (30 d of cold) completely abolished the late flowering phenotype of these transgenic lines (Fig. 1D, Fig. S1), similar to findings in the wild-type line. These results confirmed the critical role of FRI in delaying flowering time.

\section{Targeted ectopic expression of $F R I$ delays flowering time}

FLC regulates flowering time in Arabidopsis in a spatially-dependent manner, and FRI upregulates 
FLC expression (Searle et al., 2006). FRI itself encodes a large protein (MW >77 KD) that would be incapable of moving long distances, here we also found that FRI is widely distributed in different tissue. This possibility prompted us to investigate the spatial effect of $F R I$ on flowering time, thus we ectopically expressed FRI-GUS driven by various tissue-specific promoters, including $p S U C 2$ and $p$ RolC for phloem-specificity, $p K N A T 1$ for meristem-specificity, $p M L 1$ for leaf epidermis specificity, and pTobRB7 for root-specificity. GUS staining revealed that these promoters exclusively drove FRI-GUS expression in specifical tissues (Fig. 2A, Fig. S2). Furthermore, We found that all these transgenic lines of SUC2::GUS-FRI, RolC::GUS-FRI, KNATI::GUS-FRI, ML1::GUS-FRI and TobRB7::GUS-FRI showed delayed flowering (Fig. 2B-C, Fig. S3). Although targeted ectopic expression of FRI using spatially specific promoters delayed flowering, the flowering time still varied among these transgenic lines. As shown in Fig. 2B-C, the SUC2 and RolC promoters, which drove FRI expression specifically in the phloem, resulted in the greatest delays in flowering, followed by the KNAT1 promoter and $M L 1$ promoter. Expressing FRI under the control of the TobRB7 promoter also delayed flowering, but the effect was not as obvious as that of the SUC2 and RolC promoter. Vernalization treatment abolished the late flowering phenotype in these transgenic lines (Fig. S4). These data indicated that FRI could function in specific tissues, including the phloem, leaves, shoot meristem and roots, to delay flowering.

\section{Spatial expression of $F R I$ in the phloem significantly affects $F L C$ expression and its histone trimethylation level}

The transcriptional levels of $F L C$ could be markedly up-regulated by $F R I$ before vernalization (Shindo et al., 2005; Choi et al., 2011). Thus, we examined the patterns of FRI and FLC expression in these transgenic lines. As shown in Fig. 3A (upper panel), we found that the levels of FRI transcript in transgenic lines were consistent with the expression patterns driven by the spatial-specific promoters. The transcriptional level of $F L C$ was increased over 30 -fold in the leaves of the SUC2::GUS-FRI and RolC::GUS-FRI lines in comparison with those of the wild-type Col line. The FLC levels in the shoot apices of the KNAT1::GUS-FRI line and in the root tissue of the TobRB7::GUS-FRI line were also up-regulated, though to a lesser extent than in the leaves of the SUC2::GUS-FRI line (Fig. 3A, upper panel). Furthermore, we found that the FT transcript level in 
the leaves and the SOCl transcript level in the leaves and shoot apices of all these lines were markedly lower than that of wild-type plants, except the KNAT1::GUS-FRI line, which had high levels of the FT and SOC1 transcripts in the leaves (Fig. 3A, bottom panel). Vernalization treatment blocked the FLC transcription, and increased the transcription of FT and SOC1 in the leaves or shoot apices, in the transgenic lines of SUC2::GUS-FRI, and also in other transgenic lines (Fig. S5). These data suggest that Spatial expression of FRI in the phloem mainly delayed flowering by upregulating the expression of $F L C$ in the leaves.

FRI promotes FLC expression by increasing histone methylation at the $F L C$ locus, which delays flowering (Song et al., 2012). As shown in Fig. 3B, we measured the levels of histone H3Lys4 trimethylation (H3K4me3), the histone mark associated with active FLC chromatin in the leaves. Consistent with the high FLC transcript pattern in these transgenic lines, the H3K4me3 levels in the FLC genomic region were highest in the leaves of the SUC2::GUS-FRI and RolC::GUS-FRI lines, followed by the KNAT1::GUS-FRI and ML1::GUS-FRI lines. The H3K4me3 levels at the FLC locus were very low in the leaves of the TobRB7::GUS-FRI line (Fig. 3B). Furthermore, the H3K4me3 levels at the FLC locus in the root tissue of the TobRB7::GUS-FRI line were also up-regulated, though to a lesser extent than in the leaves of the SUC2::GUS-FRI line. Vernalization treatment completely suppressed the $\mathrm{H} 3 \mathrm{~K} 4 \mathrm{me} 3$ levels at the FLC locus in the leaves and roots of these transgenic lines (Fig. S6).

\section{Spatial expression of $F R I$ in the roots specifically activates $M A F 4$ and $M A F 5$ expressions and delays flowering in $F L C$-dependent pathways}

A previous study showed that the spatial expression of $F L C$ in the root did not delay flowering (Searle et al., 2006). In our current experiments, ectopic expression of FRI expression in the roots of the TobRB7::GUS-FRI line delayed flowering, and reduced levels of FT and SOC1 transcripts in the leaves (Fig. 3A), thus it is possible that FRI might activate additional genes to delay flowering in the TobRB7::GUS-FRI line. In addition to FLC, there exists five MADS gene (FLM and MAF2-5) homology to FLC in Arabidopsis genome (Ratcliffe et al., 2003; Scortecci et al., 2003; Gu et al., 2009; Kim et al., 2010). Thus, we further compared the transcript differences of FLM and MAF2-5 
in the roots of TobRB7::GUS-FRI, SUC2::GUS-FRI, 35S::GFP-FRI and wild-type lines. We found that there was no obvious change in $M A F 1, M A F 2$ or $M A F 3$ expression in the leaves and root tissue of these transgenic lines (Fig. S7A). However, the MAF4 and MAF5 transcript levels in the roots of TobRB7::GUS-FRI plants were nearly 5-fold greater than in the wild type, whereas they were not increased in the roots of other lines (Fig. 4A). These data indicate that root MAF4 and MAF5 are possible other targets of FRI for delaying flowering in Arabidopsis.

To further assess the role of root MAF4 and MAF5 in controlling flowering time, we obtained two transfer DNA (T-DNA) insertion alleles for MAF4 and MAF5 from the Arabidopsis Information Resource (TAIR), termed maf4-1 and maf5-1, respectively. These two alleles failed to produce full-length transcripts, as demonstrated by reverse transcription-PCR (Fig. S7B-C). We then crossed maf4-1 with maf5-1 to obtain the maf4/maf5-1 double mutant (Fig. S7B), and introduced TobRB7::GUS-FRI into the maf4/maf5 double mutant (termed TobRB7::GUS-FRI/maf4/maf5), and then compared the flowering times with TobRB7::GUS-FRI line (Fig. 4B-C). Under long-day conditions, knocking out MAF4 and MAF5 in the TobRB7::GUS-FRI/maf4/maf5 line dramatically reduced flowering time, in contrast to TobRB7::GUS-FRI, suggesting that MAF4 and MAF5 are required for the late flowering phenotype of TobRB7::GUS-FRI plants. We then generated transgenic TobRB7::MAF4 line and TobRB7::MAF5 line in which MAF4 or MAF5 expression was respectively driven by the root-specific TobRB7 promoter and crossed the TobRB7::MAF4 line with the TobRB7::MAF5 line (referred to as TobRB7::MAF4/TobRB7::MAF5). As shown in Fig. 4B-C and Fig. S8, in contrast to the wild type, these transgenic plants had late flowering phenotypes, and the double overexpression plants (TobRB7::MAF4/TobRB7::MAF5) flowered later than the TobRB7::MAF4 or TobRB7::MAF5 lines alone, suggesting that MAF4 and MAF5 delay flowering when they are expressed specifically in the root.

To confirm whether the late flowering phenotype in the TobRB7::GUS-FRI line depends on FLC, we crossed SUC2::GUS-FRI or TobRB7::GUS-FRI with flc-3 (referred to as SUC2::GUS-FRI/flc-3 and TobRB7::GUS-FRI/flc-3 respectively) and then compared the flowering time of these lines. As previously reported (Searle et al., 2006), the loss-of-function flc-3 line 
showed early flowering under LD or SD conditions (Fig. 9A-B). Under long-day conditions, knocking out FLC in the SUC2::GUS-FRI/flc-3 line markedly promoted flowering, resulting in a flowering time that matched that of the wild type (Fig. 4C), suggesting that FLC was mainly responsible for the late flowering time of the $S U C 2:: G U S-F R I$ line. In addition, mutation of $F L C$ in the TobRB7::GUS-FRI/flc-3 background also dramatically promoted flowering in comparison with the TobRB7::GUS-FRI line, suggesting the synergistic effect of FLC and MAF4/MAF5 in regulating flowering time in TobRB7::GUS-FRI line.

\section{Root MAF4 and MAF5 mediate FRI-dependent late flowering through antagonizing leaves FT signal}

Grafting is a useful tool for investigating the movement of long-range signals within a plant. For instance, grafting experiments demonstrated that FT moves from leaves to the shoot meristem to initiate flowering (An et al., 2004). To evaluate whether certain mobile signals exist that travel from the roots to shoot to delay flowering once $F R I$ is expressed in the root tissue, we grafted the rootstock of TobRB7::GUS-FRI with wild-type Col scion (termed TobRB7::GUS-FRI $\rightarrow$ Col). Such grafted line had a later flowering time than the control line, in which wild-type Col rootstock was grafted to wild-type $\mathrm{Col}$ scion (termed $\mathrm{Col} \rightarrow \mathrm{Col}$ ) (Fig. 5A-B, Fig. 9C-D). The $F T$ level in the leaves of TobRB7::GUS-FRI $\rightarrow \mathrm{Col}$ line was lower than that in the control $\mathrm{Col} \rightarrow \mathrm{Col}$, and the expressions of MAF4 and MAF5 were dramatically increased in the roots of TobRB7::GUS-FRI $\rightarrow$ Col plants (Fig. 5C). Similarly, in contrast to the grafted $\mathrm{Col} \rightarrow \mathrm{Col}$ line, the graft TobRB7::MAF4/TobRB7::MAF5 $\rightarrow$ Col line also exhibited delayed flowering (Fig. 5A-B, Fig. 9C-D). These data indicated that a potential mobile factor travels from the rootstock of TobRB7::GUS-FRI or TobRB7::MAF4/TobRB7::MAF5 to suppress floral initiation in the wild-type Col scion, and such mobile factor possibly interferes with the florigen $F T$ signal to reduce $F T$ expression in the leaves.

To further test the role of $F T$ in mediating the late flowering phenotype of the TobRB7::GUS-FRI line, we introduced TobRB7::GUS-FRI into the late flowering $f t-10$ mutant (termed TobRB7::GUS-FRI/ft-10). As expected, flowering was markedly delayed in 
TobRB7::GUS-FRI/ft-10 plants, as in the $f t-10$ mutant (Fig. 9A-B). The grafted TobRB7::GUS-FRI $\rightarrow f t-10$ line (TobRB7::GUS-FRI rootstock connected to $f t-10$ scion ) showed a similar late flowering time as the $f t-10$ mutant, suggesting the late flowering phenotype of the TobRB7::GUS-FRI line requires the leaf FT signal (Fig. 5 A-B, Fig. 9C-D). Furthermore, we found that the TobRB7::GUS-FRI $\rightarrow f l c-3$ line (TobRB7::GUS-FRI rootstock to the $f l c-3$ scion) flowered earlier than did the TobRB7::GUS-FRI $\rightarrow$ Col line (Fig. 5A-B, Fig. 8C-D). These data further support that a mobile signal that functions as an anti-florigen travels from the rootstock of TobRB7::GUS-FRI to delay flowering, and the presence of functional FLC in the leaves has a certain function in the late flowering phenotype of TobRB $7:: G U S-F R I$.

\section{Misexpressing FRI in the embryo delays flowering time and increases H3K4me3 level at FLC locus}

Beside the spatial effect of $F R I$ on flowering time, we also investigate the temporal effect of $F R I$ on flowering, because epigenetic modification, which is characteristic of long-term temporal effect, play the essential role in FRI-dependent flowering time (Song et al., 2012; Wigge et al., 2005). To achieve it, we investigated the effect of FRI expression at different developmental stages. We firstly generated transgenic lines in which the expression of GUS-FRI fusions was driven by two embryo-specific promoters: $L E C 2$, for early embryogenesis specificity, and $E M 1$, for seed maturation expression (Braybrook and Harada, 2008; Gaubicr et al., 1993). As shown in Fig. 6A, GUS staining was particularly strong in the embryos of these transgenic lines, suggesting that the $E M 1$ and $L E C 2$ promoters are specifically activated GUS-FRI in the embryonic tissue (Fig. 6A). We found that all of these lines had a delayed-flowering phenotype in LD conditions (Fig. 6B-C). These transgenic lines also had high levels of $F L C$ expression and $\mathrm{H} 3 \mathrm{~K} 4 \mathrm{me} 3$ modification within the FLC locus in the embryo compared with the wild type (Fig. 6D-E), suggesting that FRI expression at the embryonic stage efficiently delays flowering time, possibly through epigenetic modification of the $F L C$ locus. We also found that vernalization treatment abolished $\mathrm{H} 3 \mathrm{~K} 4 \mathrm{me} 3$ modification at the FLC locus in these transgenic lines to promote flowering, similar to the wild type (Fig. 6C-E, Fig. S10).

Misexpressing FRI at different developmental stages affect the flowering time and H3K4me3 


\section{level at FLC locus}

To determine which development status is prerequisite for FRI-dependent flowering time, we applied the estrogen-inducible system by driven FRI with the estrogen-inducible promoter (Zuo et al., 2000). We inserted the GUS-FRI fusion downstream of the lexA-binding domain, and transgenically expressed this construct (ProER8:GUS-FRI) in Arabidopsis by Agrobacterium-mediated transformation. As shown in Fig. S11, the GUS reporter gene was induced after $24 \mathrm{~h}$ of estradiol treatment in the transgenic ProER8:GUS-FRI line. We then treated the transgenic ProER8:GUS-FRI line with estradiol at different development period to see the flowering variance. We firstly tested the induction of FRI expression in the pro-embryo to post-embryonic period. Exogenous application of estradiol in the flower bud formation stage (2 days before self-pollination) markedly delayed flowering in the first generation (Fig. 7A-C). However, estradiol treatment for the flowers at the day, 1 day, 3 days, 5 days or 7 days after artificial pollination had different effects on flowering of the next generation. As shown in Fig. 7A-C, estradiol treatment at the day and one day after pollination, the progeny plants flowered very late. In contrast to the late flowering phenotype, estradiol treatment at 3 days after pollination had weak effect on flowering of the next generation, especially in the later seed formation stages. The levels of FLC transcript and the $\mathrm{H} 3 \mathrm{~K} 4 \mathrm{me} 3$ levels at the FLC locus in the progeny plants were coincide with the flowering time under LD conditions (Fig. 7D-E). These data suggest that FRI expression in the pro-embryo to post-embryonic period affects flowering time, and that estradiol induced FRI expression during gametogenesis and early embryogenesis has programed epigenetic modifications in the FLC chromatin of the next generation, but estradiol appeared not to penetrate the developing seed coat to influence flowering of the progeny plants when application of estradiol at later embryogenesis.

To further assess the contribution of FRI in maternal and paternal gametes to controlling flowering time, we treated flower buds (2 days before self-pollination) with estradiol and crossed the female parent that with or without estradiol treatment with the male parent that without or with estradiol treatment by emasculation and artificial pollination. We found that the progeny by crossing the pistil (with estradiol treatment) with the stamen (without estradiol treatment) exhibited delayed 
flowering. The progeny by crossing the pistil (without estradiol treatment) with the stamen (with estradiol treatment) also delayed flowering, though the effect of delaying flowering was greatly reduced (Fig. 7B). In addition, the FLC expression levels and the H3K4me3 levels at the FLC locus of the progeny by crossing the pistil (with estradiol treatment) with the stamen (without estradiol treatment) were higher than that of the progeny by reverse crossing (Fig. 7D-E). Our findings suggest that estradiol induced FRI expression during female gametogenesis is more inheritable and stable than that during male gametogenesis through epigenetic modifications in the FLC chromatin of the next generation.

Furthermore, estradiol treatment at the seed stage, 5 days and 7 days old seedling stages markedly delayed flowering. However, the effect of inhibiting flowering was greatly reduced when the 9 days old seedlings were treated with estradiol. Moreover, 11 days, 13 days or 15 days old seedlings did not delay flowering after estradiol treatment (Fig. 8A-B). These data indicate that flowering is only delayed if $F R I$ is expressed in the embryo or in young seedlings. We then measured FRI, FLC, FT, and SOC1 expression and H3K4me3 levels at the FLC locus in the ProER8:GUS-FRI line treated with estradiol at different growth stage. As shown in Fig. 8C and Fig. S12, the FRI expression was induced after $12 \mathrm{~h}$ of estradiol treatment in all transgenic ProER8:GUS-FRI lines of different stages. The levels of FLC transcript were high and those of FT and $S O C l$ transcript were low in the induced seedlings. However, the $F L C$ expression levels were reduced and the $F T$ and SOC1 transcript levels were increaced when the 9-15 days old seedlings treating with estradiol, compared with the younger seedlings. The H3K4me3 levels and H3K27me3 levels at the $F L C$ locus were coincide with the expression pattern of $F L C$ (Fig. 8D). Recently, a new study found that FT can suppress FLC mRNA expression and change the FLC transcription states (Chen and Penfield, 2018). In this study, the lower levels of FLC transcript and higher levels of H3K27me3 in FLC chromatin in older seedlings is probably due to the regulation of FT protein existed in the leaves. Consistent with this, even though the FT and SOC1 mRNA expression were suppressed by $F L C$ in older seedlings, but the plants showed a similar early flowering time as the non-induced seedlings. Together, these data indicate that FRI must be expressed at early development stage to ensure the epigenetic activation of FLC expression for late flowering. 


\section{Discussion}

\section{FRI targets root MAF4 and MAF5 to regulate flowering time}

For most plants, day length and environmental temperature are important flowering cues (Jaeger et al., 2007; Amasino and Michaels, 2010). Since flower development occurs at the shoot apical meristem or in the lateral meristem, but photoperiod and ambient temperature signals are perceived by the growing leaves, it is not surprising that the flowering signal is communicated among spatially separated organs in plant. FT acts both in the phloem and meristem to trigger flowering (Corbesier et al., 2007; Wigge, 2011). Misexpression of FLC from a phloem-specific promoter also represses flowering by reducing $F T$ transcript levels in the leaves, and the late flowering phenotype of such plant can be overcome by expressing FT in the same tissue (Searle et al., 2006). In our experiments, we found that FRI is globally expressed in different tissues, including leaves, meristems, phloem, and roots. The spatial expression of $F R I$ in the phloem prominently activated FLC expression and delayed flowering time. Furthermore, Our results showed that ectopic expression of FRI in the root tissue of TobRB7::GUS-FRI plants also repressed flowering, though these plants flowered earlier than did the SUC2::GUS-FRI line.

Previous studies mainly focused on the role of leaves in perceiving light or ambient signals to initiate flowering (An et al, 2004; Searle et al., 2006, Amasino and Michaels, 2010). However, in Sinapis alba, sucrose triggers the release of cytokinins from the roots during photoperiodic treatment, which is necessary for floral induction (Bernier et al., 1993). Recently, global transcriptome analysis identified 595 genes, including 18 known flowering time genes, which were differentially expressed in the root tissue under inductive long-day conditions (Bouche et al, 2016), suggesting that root tissue is integrated into the whole plant network governing flowering time. A previous study showed that ectopic expression of FLC in the root did not delay flowering (Searle $e t$ al., 2006), implying that FRI upregulates new target to delay flowering in the TobRB7::GUS-FRI line. It has been reported that FLC homologues, FLOWERING LOCUS M (FLM)/MADS AFFECTING FLOWERING 1, and MAF2-MAF5, are also associated with flowering time (Ratcliffe 
et al., 2003; Scortecci et al., 2003; Gu et al., 2009; Kim et al., 2010). Here we excluded MAFI-MAF3 as the potential targets of FRI in the TobRB7::GUS-FRI line, because their expressions were not markedly upregulated in the TobRB7::GUS-FRI line. However, two other possible targets, MAF4 and MAF5, were specifically upregulated in the roots of the TobRB7::GUS-FRI line, but not in the roots and leaves of $S U C 2:: G U S-F R I$ plants, suggesting that root MAF4 and MAF5 in the TobRB7::GUS-FRI line may function in mediating flowering.

We then observed that overexpressing MAF4 and MAF5 in the root indeed delayed flowering than the wild type, whereas mutation of MAF4 and MAF5 in the TobRB7::GUS-FRI/maf4/maf5 line promoted flowering, resulting in early flowering than in the TobRB7::GUS-FRI plants. These data support the notion that FRI targets MAF4 and MAF5 to delay flowering in the TobRB7::GUS-FRI line. Previous studies also reported that FLOWERING LOCUS C clade members act in partial redundancy in floral repression and mediate flowering responses (Gu et al., 2013). Here we found that mutation of FLC in the TobRB7::GUS-FRI line markedly promoted flowering, suggesting that $F L C$ is also required for the late-flowering phenotype of the TobRB7::GUS-FRI line, possibly via combined function of FLC and MAF4/MAF5 in regulating flowering.

\section{Spatial expression of $F R I$ or $M A F 4 / M A F 5$ in the root antagonizes the $F T$ signal in leaves to}

\section{delay flowering}

In our experiments, the transgenic expression of $F R I$ in the root activated MAF4 and MAF5 expressions and repressed the expression of FT in the leaves, and its downstream target SOCl in the shoot apex, thereby delaying flowering. These data suggest that the FT signal functions in the late flowering process by modulating the spatial expression of FRI or MAF4/MAF5 in the root. Furthermore, our grafting experiments showed that, as in TobRB7::GUS-FRI plants, the FT transcript levels in the scion leaves of TobRB7::GUS-FRI $\rightarrow$ Col was low compared to the wild-type Col. Nevertheless, we observed that the TobRB7::GUS-FRI/ft-10 plants and the grafted TobRB7:: $G U S$-FRI $\rightarrow f t-10$ plants had similar flowering times as the $f t-10$ mutant. These data suggest that systemic signals may exist that move from the rootstock to the shoot, where they possibly act downstream of $F R I$ to antagonize $F T$ expression to delay flowering. We speculate that this systemic 
signal may either be antiflorigen or a floral repressor. Antiflorigen was previously proposed to oppose florigen and was identified as a mobile signal that precisely controlled flowering time (Matsoukas, 2015). In Arabidopsis, RELATIVE OF CENTRORADIALIS (ATC) is regarded as antiflorigen, since $F T$ and $A T C$ both interact with $F D$ to affect the same downstream genes, such as AP1, but have opposite effects on their expression (Huang et al., 2012). Small non-coding RNAs, such as miR156 or miR172, may also function as antiflorigen to regulate the juvenile-to-adult and vegetative-to-reproductive phase transitions in several plant species (Wang et al., 2009; Wu et al., 2009, Skopelitis et al., 2018). Transcriptional and posttranscriptional gene silencing that are mediated by mobile signal can travel long distance from the root to the shoot through phloem (Liang et al., 2012). Deng et al. reported that an inverted-repeat RNA targets intronic regions to promote FT expression in Arabidopsis (Deng and Chua, 2015). Therefore, it is possible that the systemic signals that travel from the roots of TobRB7:GUS-FRI plants to the shoot apices are small mobile proteins or non-coding RNAs that inhibit flowering. In agreement with this, long-distance GFP silencing signal can travel from the root to the shoot, and then suppresses GFP expression in the 35S-GFP scion leaves (Liang et al., 2012). Thus, more work is needed to identify this signal and understand how it cooperates with $F T$ to accurately modulate flowering time.

\section{Expressing FRI during embryonic development and in the young seedling stage efficiently delayed flowering time}

It is reported that the seed-specific transcription factor LEAFY COTYLEDON1 ( $L E C 1)$ promotes the initial establishment of an active chromatin state at FRI-mediated FLC and activates its expression de novo in the pro-embryo (Tao et al., 2017). Both of LEC1 and LEC2 are master regulators for seeds development, $L E C 2$ directly interacts with $L E C 1$ (NF-YB9) in developing seeds, and EM1 is specifically expressed in the embryo (Boulard et al., 2018, Gaubicr et al., 1993). To investigate the temporal effect, in particular, during gametogenesis, pro-embryogenesis and post-embryogenesis, on FRI-mediates flowering time, we used an estradiol-inducible expression system, or mis-expressed FRI using embryo-specific promoters. We found that FRI delays flowering when expressed using seed-specific promoters, and that this was accompanied by high H3K4me3 levels at the $F L C$ locus and high levels of FLC expression. Furthermore, estradiol induced FRI expression 
during gametogenesis and early embryogenesis delayed flowering by epigenetic modifications in the FLC chromatin of the next generation. These results are consistent with previous study which showed that the epigenetic reprogramming of chromatin state occurred as early as the time of gametogenesis (Tao et al., 2017). Activation of FRI during female gametogenesis had more contribution to delaying flowering than activation of FRI during male gametogenesis, suggesting that matrilineal inheritance is more important than paternal inheritance in embryonic epigenetic programming at the $F L C$ locus.

We also found that estradiol treatment at the embryo stage or small seedlings of less than one-week-old markedly delayed flowering, and was accompanied by high levels of FLC transcript and H3K4me3 modification, as well as low FT and SOC1 transcript levels. However, inducing FRI expression at a later stage (i.e., more than one week after germination) did not effectively delay flowering. These data indicate that the timing of FRI expression affects flowering time; particularly, the post-embryo and young seedlings are most sensitive to FRI-mediated suppression of flowering. Consistent with our study, Sheldon et al. found that FLC activity during late embryonic development is a prerequisite for the repressive action of FLC on flowering (Sheldon et al., 2008). Furthermore, FRI maintains high levels of FLC expression in later embryonic and vegetative development (Choi et al., 2009). Reprogramming of FLC expression by FRI during embryonic and vegetative growth ensures that FT is repressed before winter so that the long-day photoperiod of spring is able to induce $F T$ activity, with flowering occurring at an optimal time.

In conclusion, here we reported the spatial and temporal effects of $F R I$ on flowering time in Arabidopsis, and found spatially expressing FRI in the root tissue, not only in the leaves or phloem, also efficiently delayed flowering. We further identified MAF4 and MAF5 in the roots acted as the novel target for FRI to delay flowering. Meanwhile, temporally expressing FRI during the pro-embryo or post-embryo stage also efficiently activated FLC and delay flowering. On the basis of our data, we propose a working model for this mechanism (Fig. 9). Before vernalization, ectopic expression of FRI in the embryo, young seedlings, in the leaves or phloem activates $F L C$, or in the roots activates MAF4 and MAF5. Activated FLC directly blocks the FT signal in the leaves to delay 
flowering. Correspondingly, activated MAF4/MAF5 in roots might induce the transcription of the gene encoding the antiflorigen-like molecule to antagonize FT expression in the leaves. During vernalization, the cold signal could be perceived by the whole plant, relieving the FT signal, and finally promoting floral initiation. Taken together, our findings provide insight into the mechanism by which FRI modulates flowering during plant development, and demonstrate that roots, and not just leaves, perceive the flowering signal to fine-tune the flowering time.

\section{Supplementary data}

Table S1. Primers used in this study.

Fig. S1. Constitutive expression of FRI delays flowering.

Fig. S2. Localization of GUS-FRI expression by GUS staining.

Fig. S3. FRI expression under the control of the SUC2, RolC, KNAT1, ML1 and TobRB7 promoters delayed flowering.

Fig. S4. Flowering phenotypes among various transgenic lines after vernalization.

Fig. S5. Quantitative RT-PCR analysis of the spatial expressions of FLC, FT and SOC1 in different tissues from different transgenic lines after vernalization.

Fig. S6. CHIP assay of the relative levels of $\mathrm{H} 3 \mathrm{~K} 4 \mathrm{me} 3$ in $F L C$ chromatin after vernalization.

Fig. S7. $M A F 1, M A F 2$ or $M A F 3$ expression in the leaves and root tissue of these transgenic lines was no obvious change.

Fig. S8. MAF4 and MAF5 expression under the control of the TobRB7 promoter delayed flowering. 
Fig. S9. The different flowering times of $f t$, flc mutants and the different grafts with TobRB7::GUS-FRI as the rootstock.

Fig. S10. Flowering phenotypes of ProLEC2::GUS-FRI and ProEM1::GUS-FRI transgenic lines.

Fig. S11. Identification of ProER8:GUS-FRI by GUS staining.

Fig. S12. Quantitative RT-PCR analysis of the expressions of FT and SOC1 in the ProER8:GUS-FRI line treated with or without estradiol.

\section{Acknowledgements}

We thank Dr. Hailong An for providing the constructs containing different promoters, and ABRC for providing the T-DNA insertion seeds. This article was supported by the National Science Foundation of China (No. 31470348), the Natural Science Foundation of Yunnan Province (NO. 2016FA015) and start-up funding from Shanghai University. X.K. was supported by the National Science Foundation of China (No. 31500221) and the 13th Five-year Informatization Plan of Chinese Academy of Sciences (No. XXH13506).

\section{References}

Abe M, Kobayashi Y, Yamamoto S, Daimon Y, Yamaguchi A, Ikeda Y, Ichinoki H, Notaguchi M, Goto K, Araki T. 2005. FD, a bZIP Protein Mediating Signals from the Floral Pathway Integrator FT at the Shoot Apex. Science 309, 1052-1056.

Amasino RM, Michaels SD. 2010. The Timing of Flowering. Plant Physiology 154, 516-520.

An H, Roussot C, Suárez-López P, Corbesier L, Vincent C, Piñeiro M, Hepworth S, Mouradov A, Justin S, Turnbull C. 2004. CONSTANS acts in the phloem to regulate a systemic signal that induces photoperiodic flowering of Arabidopsis. Development 131, 3615.

Bernier G, Havelange A, Houssa C, Petitjean A, Lejeune P. 1993. Physiological Signals That Induce Flowering. Plant Cell 5, 1147-1155.

Bouché F, D’Aloia M, Tocquin P, Lobet G, Detry N, Périlleux C. 2016. Integrating roots into a whole plant network of flowering time genes in Arabidopsis thaliana. Scientific Reports 6, 29042.

Boulard C, Thévenin J, Tranquet O, Laporte V, Lepiniec L, Dubreucq B. 2018. LEC1 (NF-YB9) directly interacts with LEC2 to control gene expression in seed. Biochim Biophys Acta 1861, 443-450. 
Braybrook SA, Harada JJ. 2008. LECs go crazy in embryo development. Trends in Plant Science 13, 624-630.

Choi J, Hyun YKang MJ, In YH, Yun JY, Lister C, Dean C, Amasino RM, Noh B, Noh YS, Choi Y. 2009. Resetting and regulation of Flowering Locus C expression during Arabidopsis reproductive development. Plant Journal 57, 918-931.

Choi K, Kim J, Hwang HJ, Kim S, Park C, Sang YK, Lee I. 2011. The FRIGIDA Complex Activates Transcription of FLC, a Strong Flowering Repressor in Arabidopsis, by Recruiting Chromatin Modification Factors. Plant Cell 23, 289-303.

Corbesier L, Vincent C, Jang S, Fornara F, Fan Q, Searle I, Giakountis A, Farrona S, Gissot L, Turnbull C, Coupland G. 2007. FT protein movement contributes to long-distance signaling in floral induction of Arabidopsis. Science 316, 1030-1033.

Deng S, Chua NH. 2015. Inverted-repeat RNAs targeting FT intronic regions promote FT expression in Arabidopsis. Plant \& Cell Physiology 56.

Gaubier P, Raynal M, Hull G, Huestis GM, Grellet F, Arenas C, Pagès M, Delseny M. 1993. Two different Em-like genes are expressed in Arabidopsis thaliana seeds during maturation. Molecular and General Genetics MGG 238, 409-418.

Geraldo N, Bäurle I, Kidou S, Hu X, Dean C. 2009. FRIGIDA Delays Flowering in Arabidopsis via a Cotranscriptional Mechanism Involving Direct Interaction with the Nuclear Cap-Binding Complex. Plant Physiology 150, 1611-1618.

Gu X, Jiang D, Wang Y, Bachmair A, He Y. 2009. Repression of the floral transition via histone H2B monoubiquitination. Plant Journal 57, 522-533.

Gu X, Le C, Wang Y, Li Z, Jiang D, Wang Y, He Y. 2013. Arabidopsis FLC clade members form flowering-repressor complexes coordinating responses to endogenous and environmental cues. Nature Communications 4, 1947.

Hu X, Kong X, Wang C, Ma L, Zhao J, Wei J, Zhang X, Loake GJ, Zhang T, Huang J. 2014. Proteasome-mediated degradation of FRIGIDA modulates flowering time in Arabidopsis during vernalization. Plant Cell 26, 4763-4781.

Huang NC, Jane WN, Chen J, Yu TS. 2012. Arabidopsis thaliana CENTRORADIALIS homologue (ATC) acts systemically to inhibit floral initiation in Arabidopsis. Plant Journal 72, 175. Jaeger KE, Graf A, Wigge PA. 2006. The control of flowering in time and space. Journal of Experimental Botany 57, 3415-3418.

Jaeger KE, Wigge PA. 2007. FT protein acts as a long-range signal in Arabidopsis. Current Biology Cb 17, 1050-1054.

Johanson U, West J, Lister C, Michaels S, Amasino R, Dean C. 2000. Molecular analysis of FRIGIDA, a major determinant of natural variation in Arabidopsis flowering time. Science 290, 344.

Kim DH, Doyle MR, Sung SB, Amasino RM. 2009. Vernalization: winter and the timing of flowering in plants. Annual Review of Cell \& Developmental Biology 25, 277. 
Kim DH, Sung S. 2010. The Plant Homeo Domain finger protein, VIN3-LIKE 2, is necessary for photoperiod-mediated epigenetic regulation of the floral repressor, MAF5. Proceedings of the National Academy of Sciences of the United States of America 107, 17029.

Kim DH, Sung S. 2013. Coordination of the vernalization response through a VIN3 and FLC gene family regulatory network in Arabidopsis. Plant Cell 25, 454-469.

Li Z, Jiang D, He YH. 2018. FRIGIDA establishes a local chromosomal environment for FLOWERING LOCUS C mRNA production. Nature Plants 4, 836-846.

Liang D, White RG, Waterhouse PM. 2012. Gene silencing in Arabidopsis spreads from the root to the shoot, through a gating barrier, by template-dependent, nonvascular, cell-to-cell movement. Plant Physiology 159, 984-1000.

Matsoukas IG. 2015. Florigens and antiflorigens: a molecular genetic understanding. Essays in Biochemistry 58, 133-149.

Michaels SD, Bezerra IC, Amasino RM. 2004. FRIGIDA-related genes are required for the winter-annual habit in Arabidopsis. Proceedings of the National Academy of Sciences of the United States of America 101, 3281-3285.

Ratcliffe OJ, Kumimoto RW, Wong BJ, Riechmann JL. 2003. Analysis of the Arabidopsis MADS AFFECTING FLOWERING gene family: MAF2 prevents vernalization by short periods of cold. Plant Cell 15, 1159-1169.

Schmitz RJ, Amasino RM. 2007. Vernalization: A model for investigating epigenetics and eukaryotic gene regulation in plants. BBA - Gene Structure and Expression 1769, 269-275.

Scortecci K, Michaels SD, Amasino RM. 2003. Genetic interactions between FLM and other flowering-time genes in Arabidopsis thaliana. Plant Molecular Biology 52, 915-922.

Searle I, He Y, Turck F, Vincent C, Fornara F, Kröber S, Amasino RA, Coupland G. 2006. The transcription factor FLC confers a flowering response to vernalization by repressing meristem competence and systemic signaling in Arabidopsis. Genes Development 20, 898-912.

Sheldon CC, Hills MJ, Lister C, Dean C, Dennis ES, Peacock WJ. 2008. Resetting of FLOWERING LOCUS C expression after epigenetic repression by vernalization. Proceedings of the National Academy of Sciences of the United States of America 105, 2214-2219.

Shindo C, Aranzana MJ, Lister C, Baxter C, Nicholls C, Nordborg M, Dean C. 2005. Role of FRIGIDA and FLOWERING LOCUS $\mathrm{C}$ in determining variation in flowering time of Arabidopsis. Plant Physiology 138, 1163-1173.

Shindo C, Lister C, Crevillen P, Nordborg M, Dean C. 2006. Variation in the epigenetic silencing of FLC contributesto natural variationin Arabidopsis vernalization response. Genes Development 20 , 3079-3083.

Skopelitis DS, Hill K, Klesen S, Marco CF, Born PV, Chitwood DH, Timmermans MCP. 2018. Gating of miRNA movement at defined cell-cell interfaces governs their impact as positional signals. Nature Communications 9, 3107.

Song J, Angel A, Howard M, Dean C. 2012. Vernalization- a cold-induced epigenetic switch. 
Journal of Cell Science 125, 3723-3731.

Tao Z, Shen L, Gu X, Wang Y, Yu H, He Y. 2017. Embryonic epigenetic reprogramming by a pioneer transcription factor in plants. Nature 551, 124-128.

Turnbull CG, Booker JP, Leyser HM. 2002. Micrografting techniques for testing long-distance signalling in Arabidopsis. Plant Journal 32, 255-262.

Wang JW, Czech B, Weigel D. 2009. miR156-regulated SPL transcription factors define an endogenous flowering pathway in Arabidopsis thaliana. Cell 138, 738-749.

Wigge PA. 2011. FT, a mobile developmental signal in plants. Current biology 21, 374-378.

Wigge PA, Min CK, Jaeger KE, Busch W, Schmid M, Lohmann JU, Weigel D. 2005.

Integration of spatial and temporal information during floral induction in Arabidopsis. Science 309, 1056-1059.

Wu G, Park MY, Conway SR, Wang JW, Weigel D, Poethig RS. 2009. The sequential action of miR156 and miR172 regulates developmental timing in Arabidopsis. Cell 138, 750-759.

Yoo SD, Cho YH, Sheen J. 2007. Arabidopsis mesophyll protoplasts: a versatile cell system for transient gene expression analysis. Nature Protocols 2, 1565-1572.

Zuo J, Niu QW, Chua NH. 2000. An estrogen receptor $\square$ based transactivator XVE mediates highly inducible gene expression in transgenic plants. Plant Journal 24, 265-273. 


\section{Figure Legends}

\section{Fig. 1. Constitutive expression of FRI delays flowering.}

(A) Histochemical analysis of FRI expression in the FRI::GUS-FRI lines. FRI expression was indicated by GUS staining of the whole embryo tissue and the seedlings ( $a$, bar=100 $\mu$ m; b, bar=0.2 $\mathrm{cm}$; c, bar=0.5 cm). Strong GUS staining was observed in the leaves $(\mathrm{d}$, bar=0.5 cm), meristem $(\mathrm{e}$, bar=0.05 cm), root (f, bar=0.125 cm), and phloem $(\mathrm{g}$, bar=0.01 cm).

(B) Localization of FRI in plants. The lower leaf epidermis was peeled from transgenic 35S:GFP-FRI plants and GFP fluorescence was observed in the mesophyll cells (upper panel, bar=10 $\mu \mathrm{m})$. The 35S:FRI-GFP construct was transiently expressed in Arabidopsis protoplasts (bottom panel, bar=2 $\mu \mathrm{m}$ ).

(C) Constitutive expression of FRI in the transgenic 35S:GFP-FRI lines resulted in late flowering. Immunoblot analysis of GFP-FRI expression in the wild-type Col line and three individual transgenic 35S:FRI-GFP lines using anti-GFP antibody (upper panel). The flowering phenotype of Col and three individual lines under long-day conditions (bottom panel).

(D) Flowering time as indicated by the total leaf number in plants grown under long-day conditions (LD), short-day conditions (SD) or with $30 \mathrm{~d}$ of vernalization treatment at $4{ }^{\circ} \mathrm{C}$ (VER). The $\mathrm{x}$ axis denotes total leaf number. Each dot represents a plant. For each line, 20 plants were scored.

Fig. 2. Modulating flowering time by expressing GUS-FRI in specific tissues. 
(A) Localization of GUS-FRI expression by GUS staining. GUS staining showed GUS-FRI expression in the SUC2::GUS-FRI, RolC::GUS-FRI, KNAT1::GUS-FRI, ML1::GUS-FRI and TobRB7::GUS-FRI transgenic lines. GUS staining was observed in the whole seedling (left panel; bar $=0.5 \mathrm{~cm}$ ), the leaves (middle panel; bar=0.125 $\mathrm{cm}$ ), the root (right panel; bar=0.5 $\mathrm{cm}$ ), or meristem (right panel; bar=0.05 cm). GUS staining for the TobRB7::GUS-FRI line is only presented in the root.

(B) Variation in flowering time among various transgenic lines. All of these transgenic lines were grown under long-day conditions, and the flowering phenotypes were presented at 60 days after seed germination.

(C) Quantitative flowering time among various transgenic lines as indicated by the total leaf number under long-day conditions without vernalization (NV) or with vernalization treatment at $4^{\circ} \mathrm{C}(\mathrm{VER})$. The $\mathrm{x}$ axis denotes total leaf number. Each dot represents a plant.for each line, 20 plants were scored.

Fig. 3. FRI effectively promotes the levels of $F L C$ transcript and represses $F T$ and SOCI expression when expressed in the phloem and leaves.

(A) Quantitative RT-PCR analysis of the spatial expressions of FRI, FLC, FT and SOC1 in different tissues from different transgenic lines. Ten-day-old seedlings grown under LD conditions. The beta-tubulin gene $(T U B 2)$ was amplified as an internal control. Values are means \pm SD of three biological replicates. Bars with different letters indicate significant differences among different transgenic lines within the same tissue ( $p<0.05$, ANOVA followed by Tukey's post hoc test).

(B) CHIP-qPCR analysis of the relative levels of H3K4me3 in FLC chromatin. DNA fragments were obtained from the leaves (upper panel) and roots (bottom panel) of ten-day-old plants by CHIP using H3K4me3 antibody, and the amounts of DNA fragments after ChIP were quantified and subsequently normalized to an internal control (AGAMOUS). The primer pairs used in the PCR are shown as bars below FLC. Exons are shown as black boxes and introns as black lines. Data are means \pm SD of triplicate experiments.

Fig. 4. MAF4 and MAF5 are specifically activated in the roots of TobRB7::GUS-FRI line. 
(A) Quantitative RT-PCR analysis of the spatial expressions of MAF4 and MAF5 in different tissues from different transgenic lines. Ten-day-old seedlings grown under LD conditions. The beta-tubulin gene (TUB2) was amplified as an internal control. Values are means \pm SD of three biological replicates. Bars with different letters indicate significant differences among different transgenic lines within the same tissue ( $p<0.05$, ANOVA followed by Tukey's post hoc test).

(B) Effects of overexpressing MAF4 and MAF5 in the root and loss of function of MAF4 and MAF5 in TobRB7::GUS-FRI line on flowering time. The plants were grown under long-day (LD) conditions, and the flowering phenotype is presented.

(C) Quantitative flowering time among various transgenic lines and mutants as indicated by the total leaf number under long-day conditions. The $\mathrm{x}$ axis denotes total leaf number. Each dot represents a plant.for each line, 20 plants were scored.

Fig. 5. Variation in flowering time among different grafts with TobRB7::GUS-FRI as the rootstock.

(A) Flowering time and phenotypes (B) among different grafts with TobRB7::GUS-FRI or TobRB7::MAF4/ TobRB7::MAF5 as the rootstock. The flowering time under LD conditions is given as the total leaf number at the time of flowering, and the flowering phenotypes were presented at 40 days after seed germination. Data are means \pm SD of three replicates $(n=12)$.

(C) Quantitative RT-PCR analysis of the expressions of MAF4, MAF5 and FT in different tissues from different grafts. Ten-day-old seedlings grown under LD conditions. The beta-tubulin gene (TUB2) was amplified as an internal control. Values are means \pm SD of three biological replicates. Bars with different letters indicate significant differences among different transgenic lines within the same tissue ( $p<0.05$, ANOVA followed by Tukey's post hoc test).

Fig. 6. Modulating flowering time by expressing GUS-FRI driven by seed-specific promoters. (A) Localization of GUS-FRI expression by GUS staining. GUS staining showed that FRI is specifically expressed in the seed of ProLEC2::GUS-FRI and ProEM1::GUS-FRI transgenic lines (bar=100 $\mu \mathrm{m}$ ).

(B) Phenotypes and (C) flowering time of ProLEC2::GUS-FRI and ProEM1::GUS-FRI transgenic 
lines. The flowering phenotypes were presented at 40 days after seed germination. The flowering time is given as the total leaf number at the time of flowering under long-day conditions without vernalization (NV) or with vernalization treatment (VER). Data are means \pm SD of three replicates. For each line, 20 plants were scored. Bars with different letters are significantly different at $p<0.05$. (D) Quantitative RT-PCR analysis of the expressions of FLC in different transgenic lines. Ten-day-old seedlings grown under LD conditions without vernalization (NV) or with $30 \mathrm{~d}$ of vernalization treatment at $4^{\circ} \mathrm{C}$ (VER). The beta-tubulin gene (TUB2) was amplified as an internal control. Values are means \pm SD of three biological replicates. Bars with different letters indicate significant differences among different transgenic lines $(p<0.05)$.

(E) CHIP-qPCR analysis of the relative levels of H3K4me3 in FLC chromatin. DNA fragments were obtained from ten-day-old seedlings with or without vernalization (VER or NV) by CHIP using H3K4me3 antibody, and the amounts of DNA fragments after ChIP were quantified and subsequently normalized to an internal control (AGAMOUS). The primer pairs used in the PCR are shown as bars below FLC. Exons are shown as black boxes and introns as black lines. Data are means \pm SD of triplicate experiments.

Fig. 7. Modulating flowering time by expressing GUS-FRI in inducible ProER8:GUS-FRI line during pro-embryo to post-embryonic stage

(A) Estradiol induction in different embryonic development stages of ProER8:GUS-FRI line. Estradiol was applied to parent plant during reproductive development, from flower to seed formation. Seeds were harvested from mother plants after maturity. bar=2 $\mathrm{mm}$.

(B) Estradiol induction in pistil or Stamen of ProER8:GUS-FRI line before pollination. Pistil with estradiol-treated was crossed with stamen without estradiol-treated, or Pistil without estradiol-treated was crossed with stamen with estradiol-treated. Seeds were harvested from mother plants after maturity. bar=2 $\mathrm{mm}$.

(C) Flowering time of progeny after parent plants being treated with estradiol at the pro-embryo to post-embryonic stage. The flowering time under LD conditions is given as the total leaf number at the time of flowering. Data are means \pm SD of three replicates. For each line, 20 plants were scored. (D) Quantitative RT-PCR analysis of the expressions of FLC in the progeny. Ten-day-old seedlings 
grown under LD conditions. The expressions of $F L C$ were measured in the leaves of different progeny plants. The beta-tubulin gene (TUB2) was amplified as an internal control. Values are means \pm SD of three biological replicates. Bars with different letters indicate significant differences among progeny plants after different treatments to the parent plants $(p<0.05)$.

(E) CHIP-qPCR analysis of the relative levels of H3K4me3 in FLC chromatin of transgenic ProER8:GUS-FRI line among different progeny plants. Ten-day-old seedlings grown under LD conditions were obtained by CHIP using H3K4me3 antibody, and the amounts of DNA fragments after ChIP were quantified and subsequently normalized to an internal control (AGAMOUS). The primer pairs used in the PCR are shown as bars below FLC. Exons are shown as blue boxes and introns as black lines. Data are means \pm SD of triplicate experiments.

Fig. 8. Modulating flowering time by expressing GUS-FRI in inducible ProER8:GUS-FRI line in young seedlings.

(A) Flowering time and (B) phenotypes of transgenic ProER8:GUS-FRI line treated with estradiol at the embryonic stage or 5, 7, 9, 11, 13, 15 days after germination. The flowering time under LD conditions is given as the total leaf number at the time of flowering, and the flowering phenotypes were presented at 40 days after seed germination. Data are means \pm SD of three replicates. For each line, 20 plants were scored.

(C) Quantitative RT-PCR analysis of the expressions of FRI and FLC in the ProER8:GUS-FRI line treated with or without estradiol at the embryonic stage or 5, 7, 9, 11, 13, 15 days after germination. The seeds or seedlings grown under LD conditions were harvested 2 days after treated with or without estradiol. The beta-tubulin gene (TUB2) was amplified as an internal control. Values are means \pm SD of three biological replicates. Bars with different letters indicate significant differences among different treatments $(p<0.05)$.

(D) CHIP-qPCR analysis of the relative levels of H3K4me3 and H3K27me3 in FLC chromatin of transgenic ProER8:GUS-FRI line treated with estradiol. The seeds or seedlings grown under LD conditions were harvested 5 days after treated with or without estradiol. DNA fragments were obtained from the seeds or seedlings by CHIP using H3K4me3 or H3K27me3 antibody, and the amounts of DNA fragments after ChIP were quantified and subsequently normalized to an internal 
control (AGAMOUS). The primer pairs used in the PCR are shown as bars below FLC. Exons are shown as black boxes and introns as black lines. Data are means \pm SD of triplicate experiments.

Fig. 9. Proposed model in which the spatial-temporal expression of FRI modulates flowering time in Arabidopsis thaliana.

Before vernalization, FRI expression in the phloem or leaves could efficiently increase the $F L C$ transcripts to suppress the in-situ $F T$ signal and delay flowering. Expression of $F R I$ in the root could activate MAF4 and MAF5, and possibly generate a certain mobile silencing signal, like anti-florigen-like factor (marked with a red circle), that impairs the $F T$ signal in the leaves and finally delays flowering. During vernalization, the cold signal could be perceived by the leaves and root, triggering degradation of $F R I$, which interferes with FT activity (marked with a blue circle) and subsequently promotes flowering.

\section{Figures}

Figure 1

A

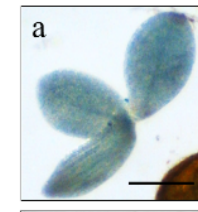

b
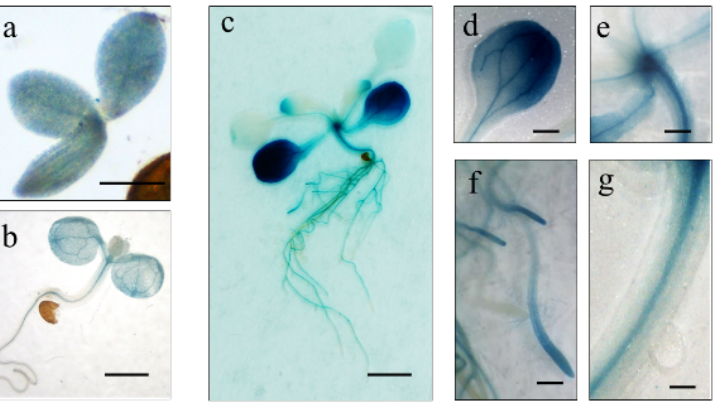

$\mathrm{B}$
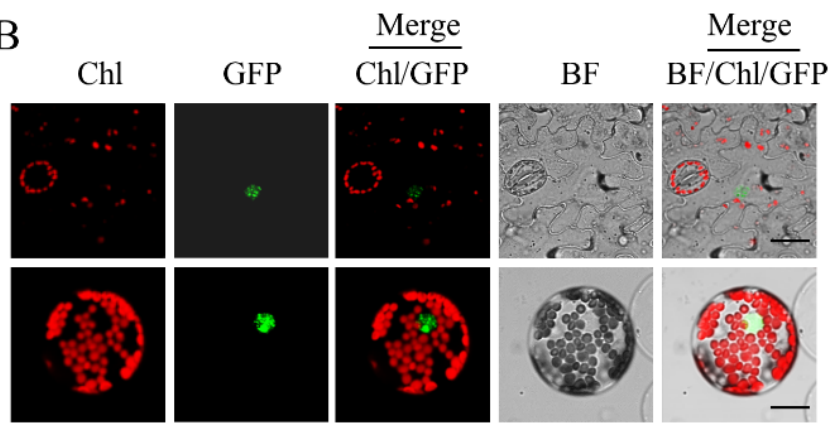
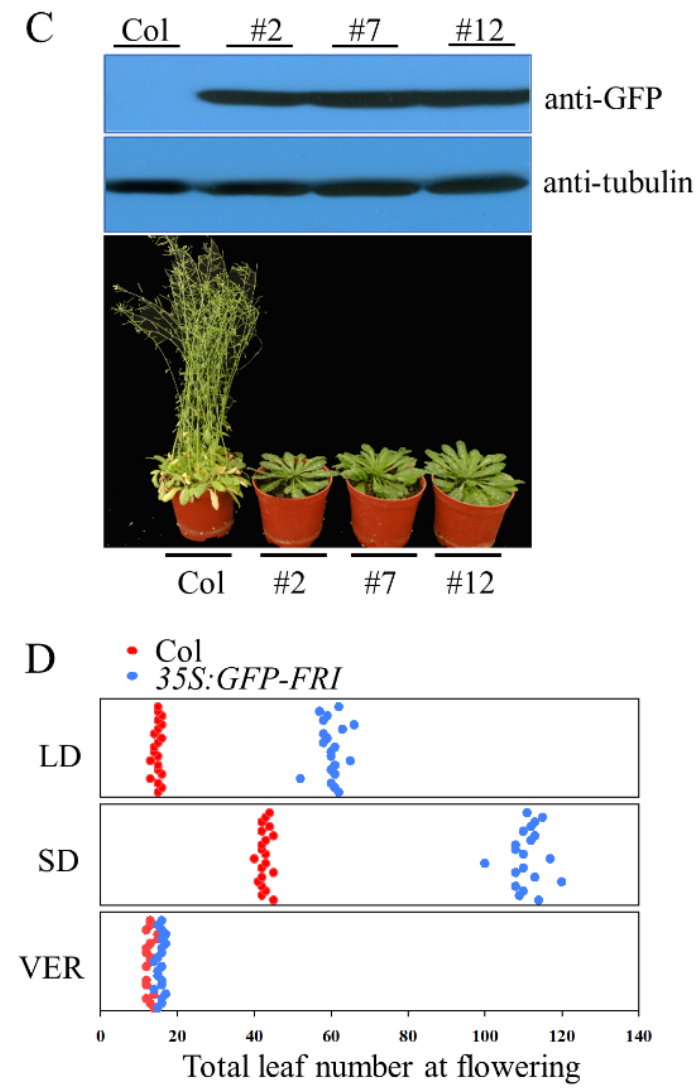
bioRxiv preprint doi: https://doi.org/10.1101/467613; this version posted November 11,2018 . The copyright holder for this preprint (which was not certified by peer review) is the author/funder. All rights reserved. No reuse allowed without permission.

Figure 2

A

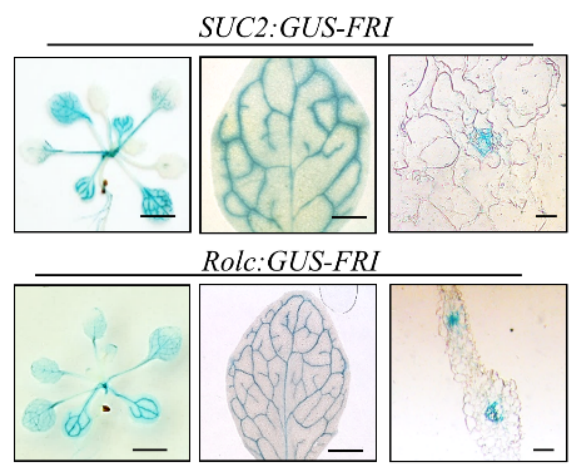

KNATI ::GUS-FRI
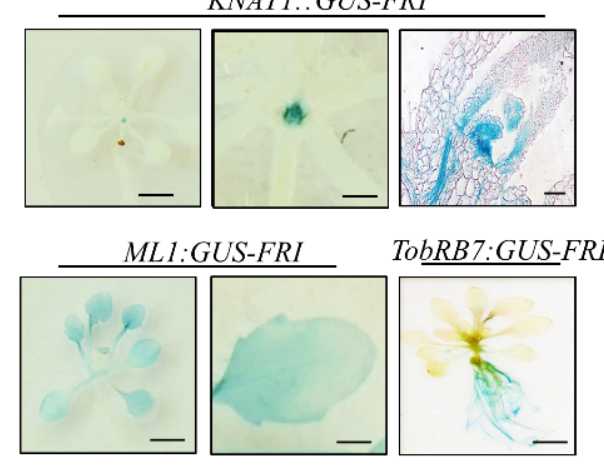

B

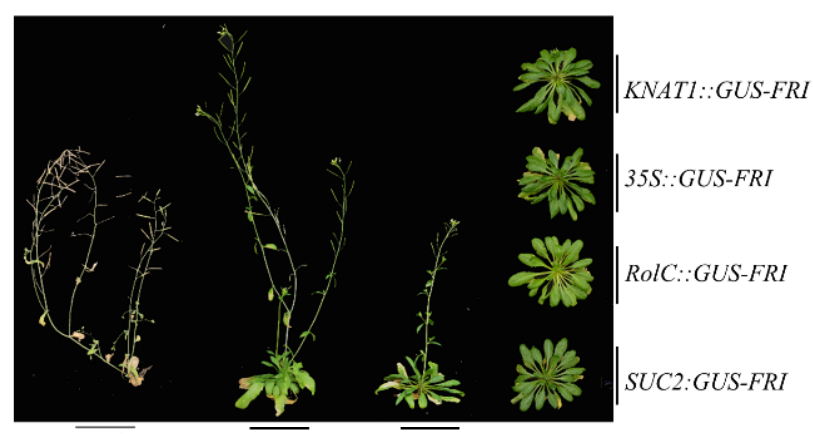

$\mathrm{C}$

$\overline{\mathrm{Col}}$ TobRB7: $\overline{G U S-F R I}$ MLI::GUS-FRI

$\mathrm{C} \quad \cdot \mathrm{Col}$

:TobRB7::GUS-FRI

- MLI::GUS-FRI

- KNAT1 :GUS-FRI

- RolC::GUS-FRI

- SUC2:GUS-FR

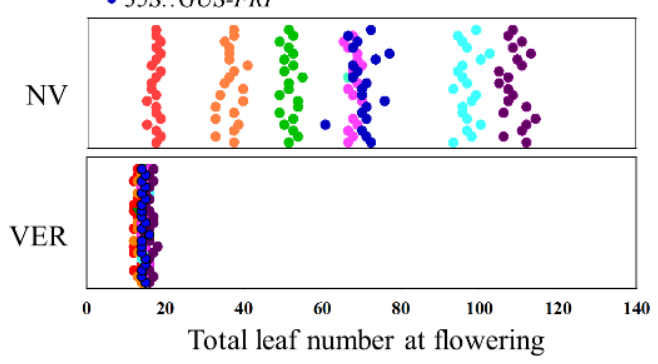


Figure 3
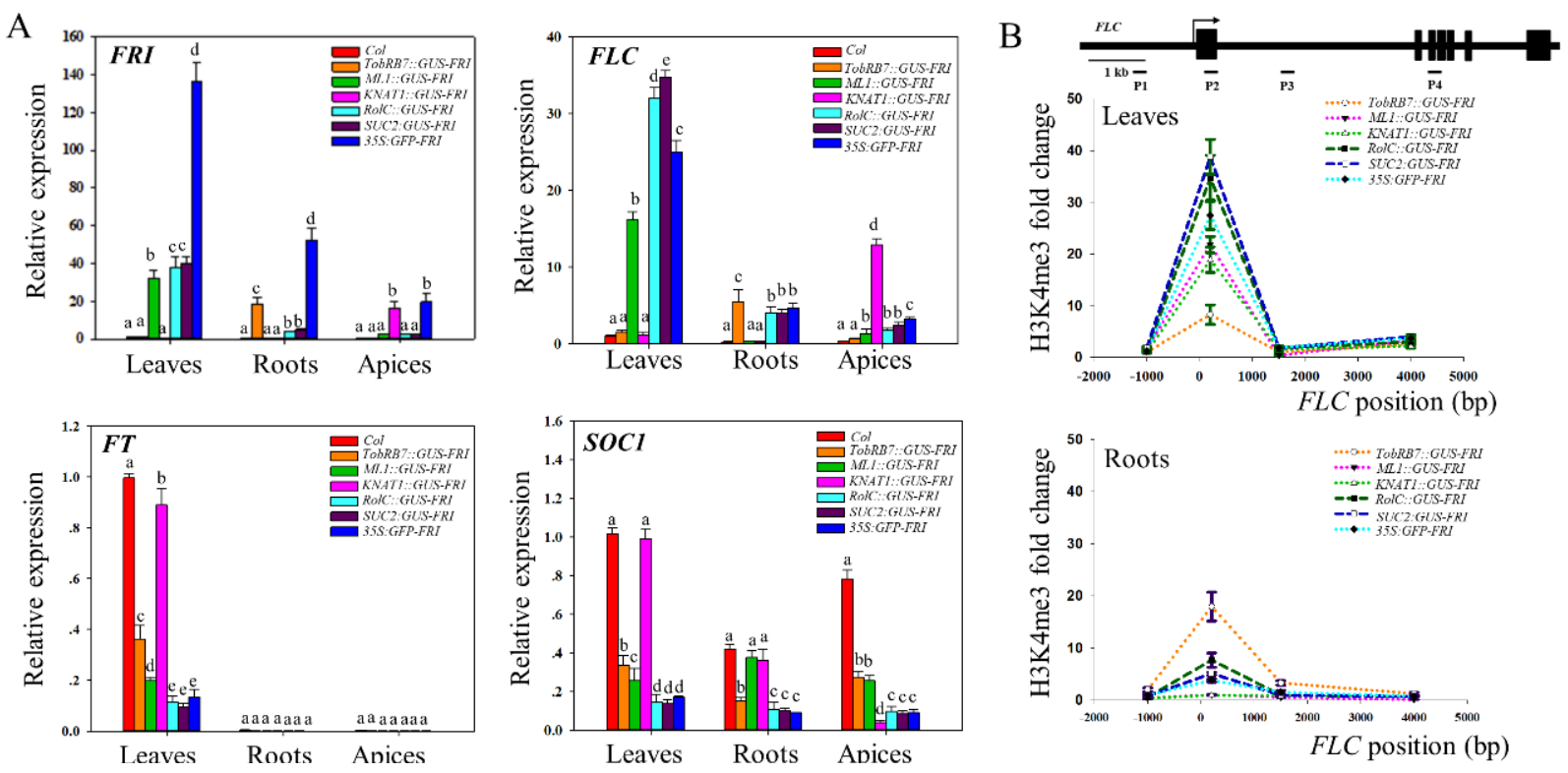


\section{Figure 4}
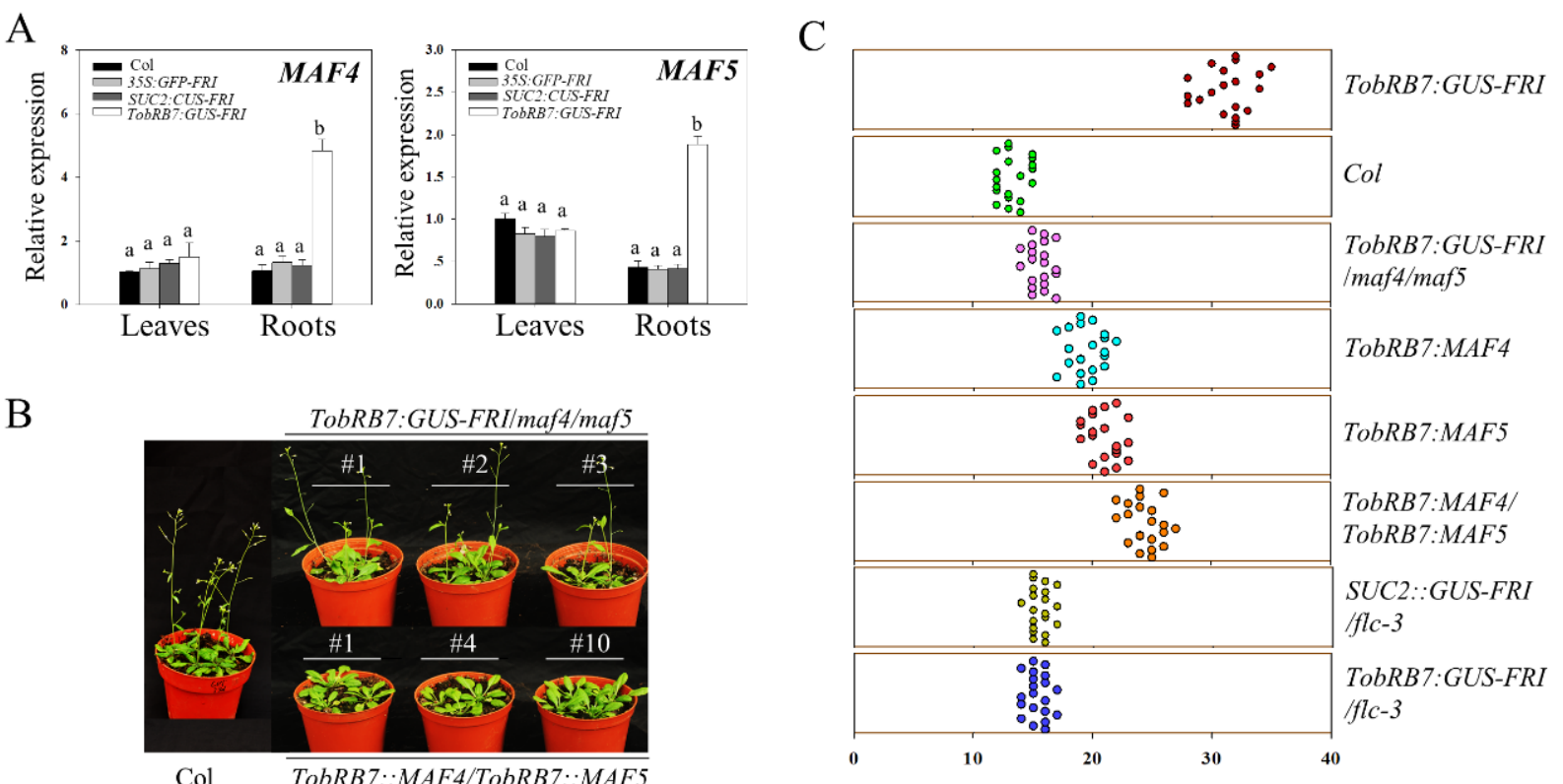

Col TobRB7::MAF4/TobRB7::MAF5

Total leaf number at flowering 


\section{Figure 5}

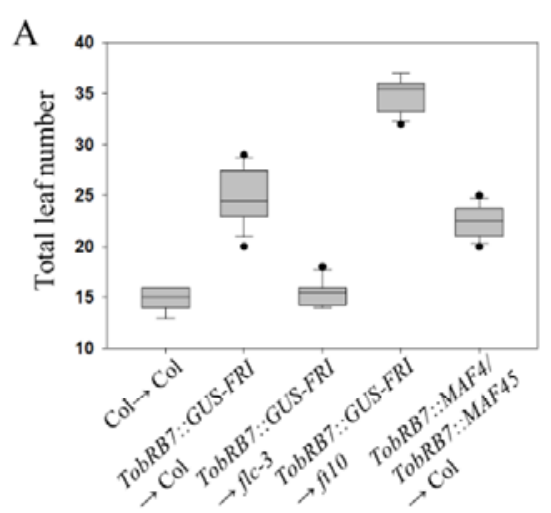

B
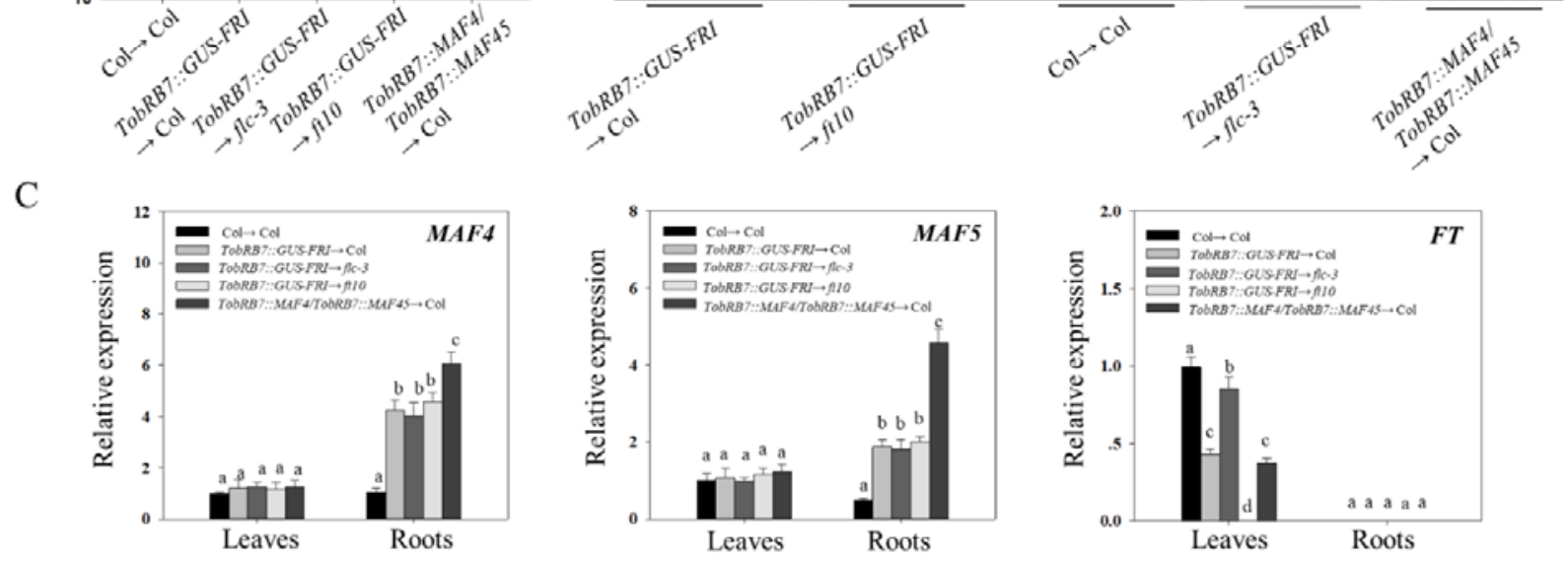


\section{Figure 6}

A

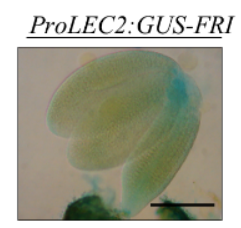

ProEMI:GUS-FRI

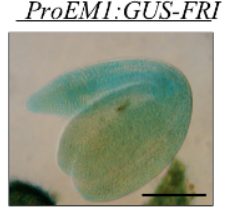

B

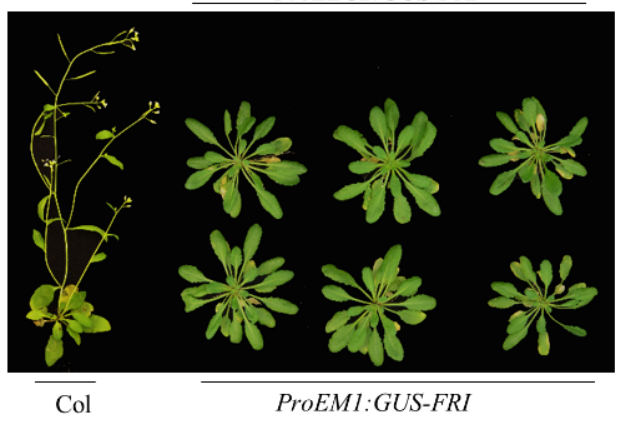

$\mathrm{C}$

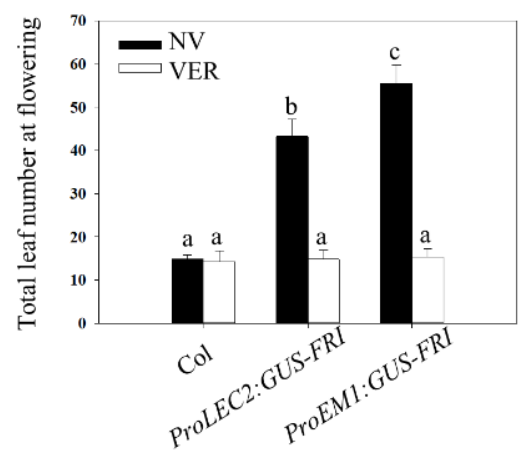

D

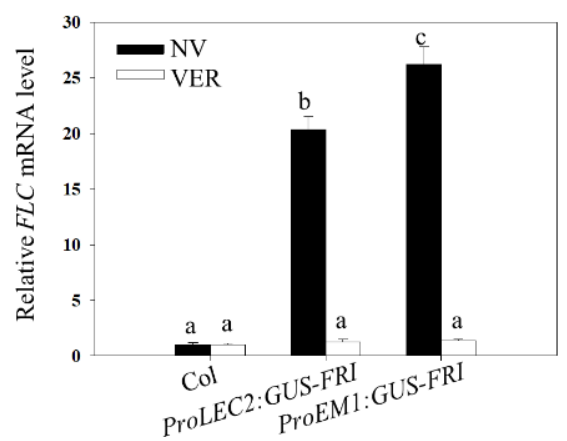

E

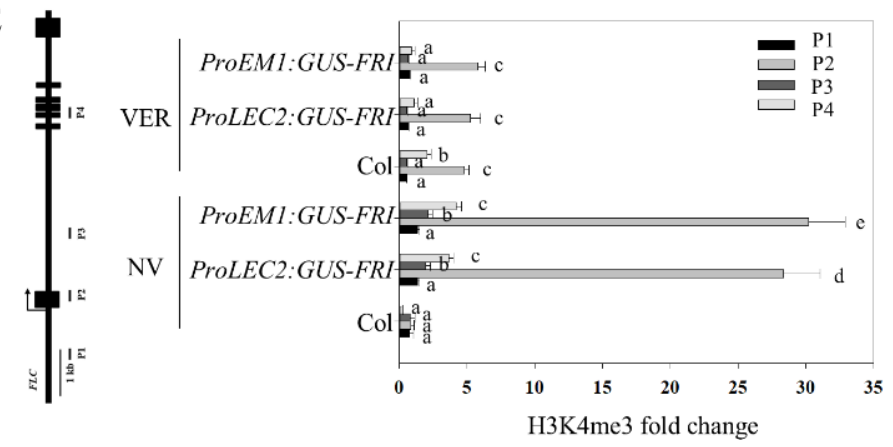


Figure 7

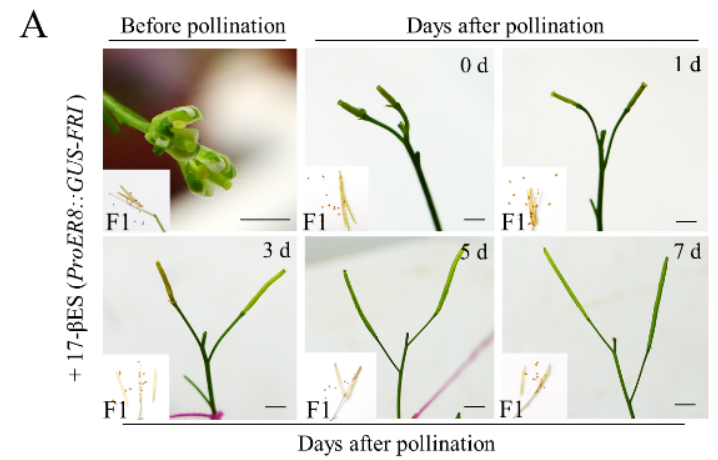

B

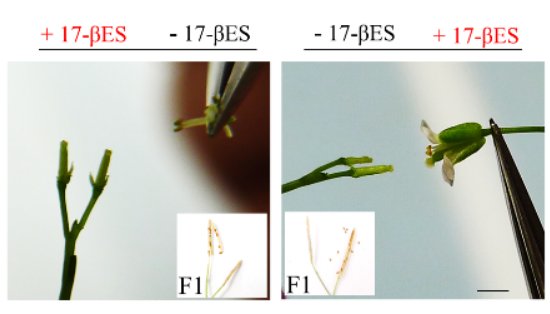

$\mathrm{D}$
$\mathrm{C}$
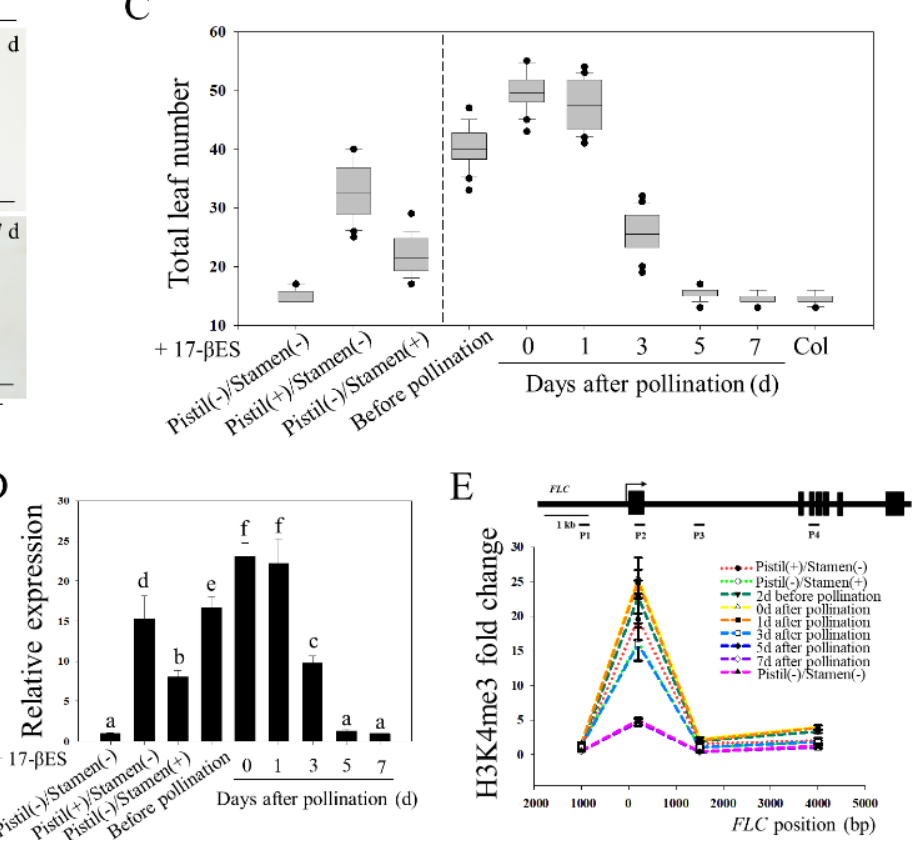


\section{Figure 8}

A

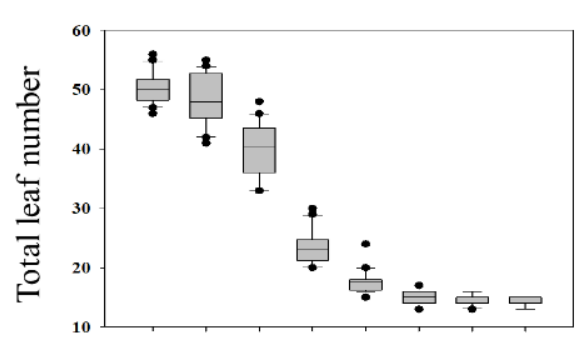

$+17-\beta E S \quad 0 d \quad 5 d \quad 7 d \quad 9 d 11 \mathrm{~d} 13 \mathrm{~d} 15 \mathrm{~d}$ Col

C
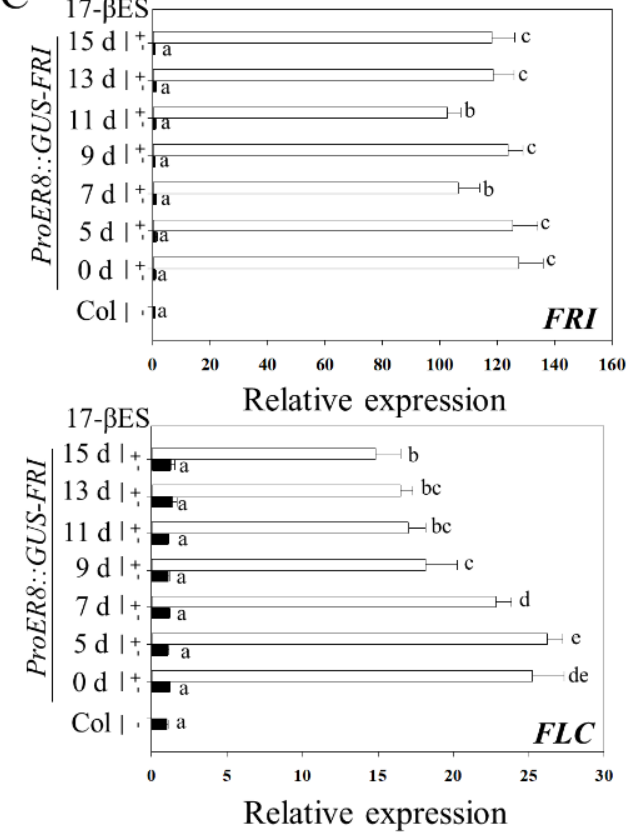

B

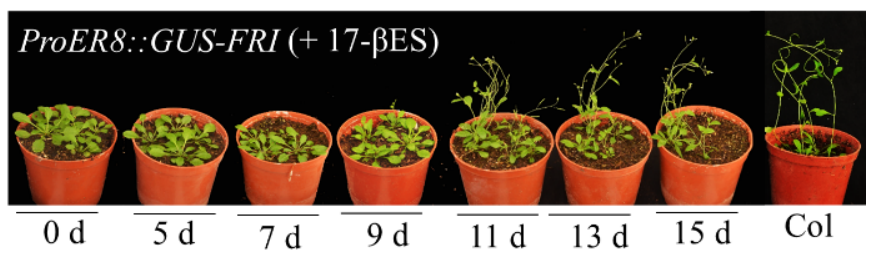

D

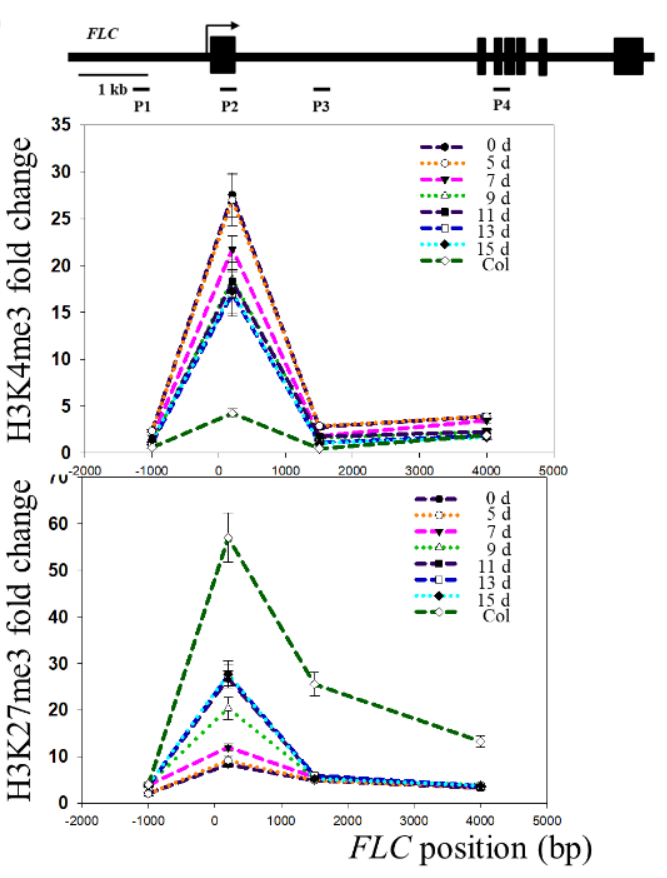




\section{Figure 9}

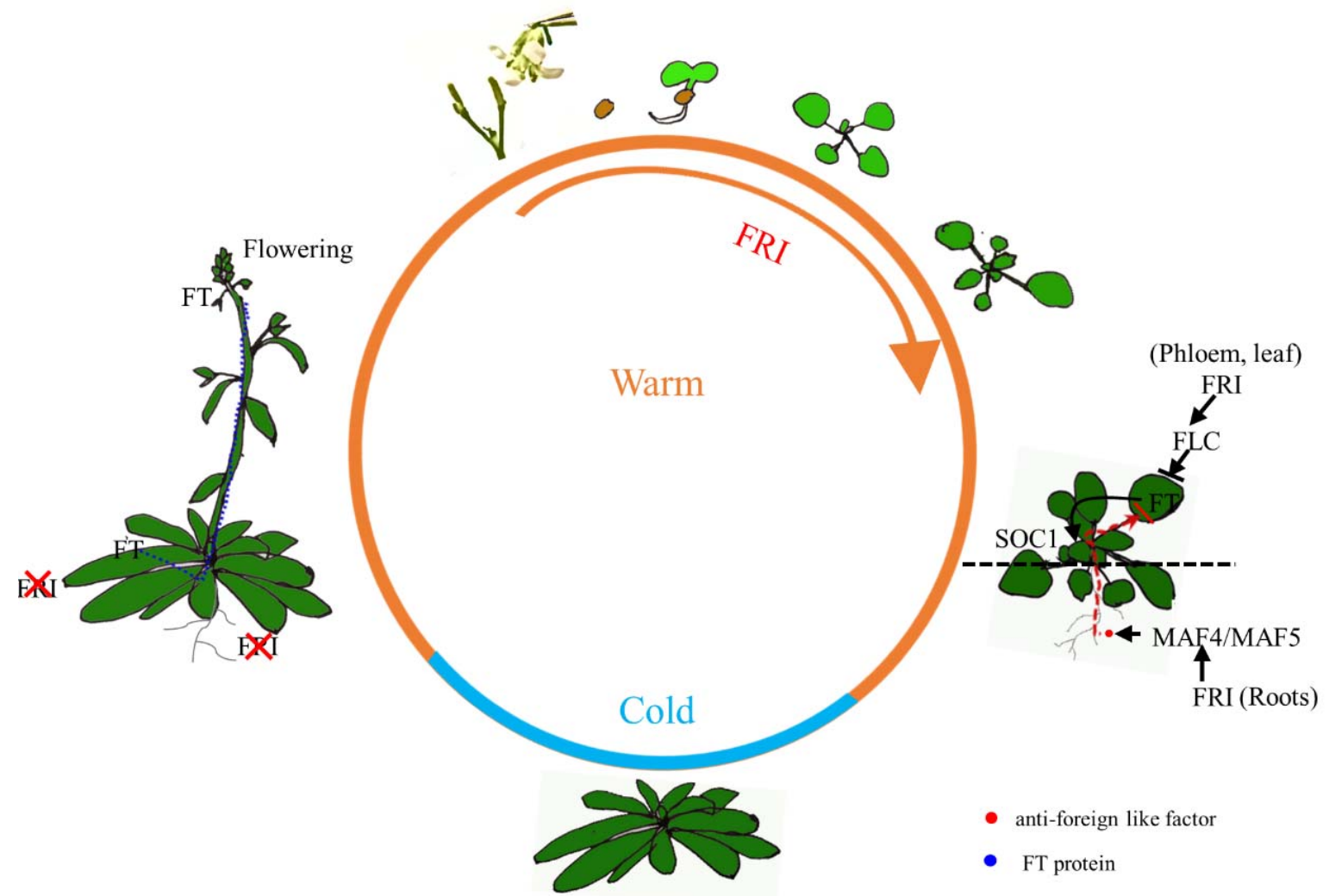

Vernalization (FLC silencing) 
bioRxiv preprint doi: https://doi.org/10.1101/467613; this version posted November 11,2018 . The copyright holder for this preprint (which was not certified by peer review) is the author/funder. All rights reserved. No reuse allowed without permission. 


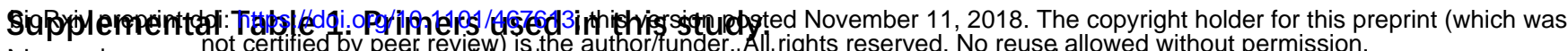
Note that sequences underlined indicate the restriction enzyme recognize sequences

\section{Transgenic plant}

Gene name

Primers $\left(5^{\prime}->3^{\prime}\right)$

FRI promoter

F: CGAGGCCT TTCTCGGCGGTCTTCAGATT

R: CGACCGGT GAGATTGCGGCGAAGAAA

SUC2 promoter

F: CGAGGCCTATTAATTTCACACACCAAGT

R: CGACCGGTATTTGACAAACCAAGAAAG

RolC promoter

F: CGAGGCCT CGGCGTCGGAAACTGGCGCC

R: CGACCGGT CATAACTCGAAGCATCCAAA

ML1 promoter

F: CGA GGCCTAAGCTTATCAAAGAAAAAAC

R: CGAAGCTT GGATTCAGGGAGTT

KNAT1 promoter F: CGAGGCCT CTTTTTGATCTAGAGCCCTA

R: CGACCGGTCCAGATGAGTAAAGATTTGA

ToRB7 promoter F: CGAGGCCT CCCCTTATTGTACTTCAATT

R: CGACCGGTGGTTTTCCAAGTTTCACATA

LEC2 promoter F: CGAGGCCTATATGTAGCTATGCAATCCAAGG

R: CGACCGGT TTTTCCCGGAGAGAGAGAGAGAG

EM1 promoter F: CGAGGCCTCGACTTCAGGAGAGGTAAGACTC

R: CGACCGGT CACGAACGATATGTAATTAAG

FRI CDS F: CGGAATTCATGTCCAATTATCCACCGAC

R: CGGGATCC CTATTTGGGGTCTAATGATGAG

GUS CDS F: CGACCGGTATGTTACGTCCTGTAGAAACCCCAAC

R: CGGAATTC TTGTTTGCCTCCCTGCTGCGGTT

MAF4 CDS F: CGGAATTC ATGGGAAGAAGAAAAGTAGAGATC

R:CG GGATCC TTACTTGAGAAGCAGGAGAGTC

MAF5 CDS F: CG GAATTC GATCTTGAAGACAAAACTCAGG

R:CGGGATCC TTACTTGAGAAGCGGGAGAGT

qRT-PCR

FRI

FLC

FT

SOC1

MAF1

MAF2

MAF3

MAF4

MAF5

ACTIN2

\section{CHIP}

FLC-P1

FLC-P2

FLC-P3

FLC-P4
F:TGGAGGAAAAGGTTGATGACTGA

R:GGACAAGAAACTGTGACCGCTTC

F:TGGAGGAAAAGGTTGATGACTGA

R:GGACAAGAAACTGTGACCGCTTC

F:CAACCCTCACCTCCGAGAATAT

R:TGCCAAAGGTTGTTCCAGTTGT

F:CTTCTAAACGTAAACTCTTGGGAGAAG

R:CCTCGATTGAGCATGTTCCTATG

F:GGAAAGAATACGTTGCTGGCAACA

R:CCGTTGATGATGGTGGCTAATTGA

F:CGAAATACATCATGCTGATGAACTTG

R:GCTTTGGACTATTTCTAGTAACTCTTTGA

F:GGAAATAAAGGTAAAACAAAACGAAGCTCTT

R:GAACTCTGATATTTGTCTACTAAGGTACA

F:GATGGGGAAGATGAAGAAGTCTGT

R:AGTCTCCGGTGGCTTGTTGT

F:GAAACAGGGGATGAAAGAGCAGTA

R:TGGGCTGTGGCCAGAGCTAT

F:GCTGAGAGATTCAGATGCCCA

R:GTGGATTCCAGCAGCTTCCAT

F:GCTGATACAAGCATTTCACCAAA

R:CTTAAATGTCCACACATATGGCAAT

F:CGACTTGAACCCAAACCTGA

R:GGATGCGTCACAGAGAACAG

F:TAGATTTGCCTCATATTTATGTGATTG

R:ATGAAGACAAGTGTTGTGGGAT

F:AGAACAACCGTGCTGCTTTT

R:TGTGTGCAAGCTCGTTAAGC 


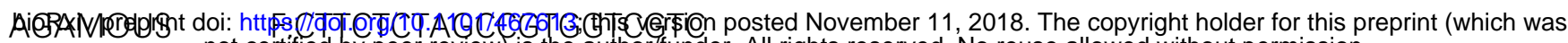
not ceptified py peer review) is the authorffynder. All rights reserved. No reuse allowed without permission.

\section{Genotype}

flc-3 mutant

TCATGCGGTACACGTGGCAA

TCGCCGGAGGAGAAGCTGTA

$\mathrm{ft}-10$ mutant

GGTGGAGAAGACCTCAGGAAC

TTTTGGGAGACAAATTGATGC

maf4-1 mutant TGATTACATATGCATGCGACC

TGAGTTATTGGGTCTCATGGG

maf5-1 mutant TTTTCAGTTGTCCAATTTGGC

LB PRIMER ATTTTGCCGATTTCGGAAC 